\title{
Closure Theorems Without Seminormality Conditions ${ }^{1}$
}

\author{
L. Cesari ${ }^{2}$ and M. B. Suryanarayana ${ }^{3}$
}

\begin{abstract}
The authors give a variety of conditions under which there is no need to explicitly require seminormality conditions in closure and lower closure theorems, and corresponding lower semicontinuity theorems. Both Lipschitz-type conditions and growth-type conditions are taken into consideration in classes $L_{p}$ and $L_{\infty}$.
\end{abstract}

Key Words. Closure theorems, semicontinuity, seminormality, Lipschitz condition, Mayer problems, Lagrange problems.

\section{Introduction}

In this paper, we state and prove closure, lower closure, and semicontinuity theorems, based on sole convexity requirements and analytical properties of the given functions, as Lipschitz-type, or growth-type conditions. In our theorems we are not using, therefore, those seminormality conditions which in Mayer and Lagrange problems are usually expressed in terms of Kuratowski upper semicontinuity of the relevant sets, and analogous variants as property (Q) (Ref. 1).

The analytical conditions we take into consideration (F-type, G-type, H-type conditions) will be listed in Section 4. These conditions are known to have practical significance. They have a bearing on the problem under consideration through a simple function-theoretical property, which we denote as property (D) (Section 3). Closure theorems for abstract Mayer problems are given in Sections 6 and 7, lower closure theorems for Lagrange problems in Sections 8 and 9, and lower semicontinuity theorems for free problems of the calculus of variations in Section 10.

1 This research was done in the framework of AFOSR Research Project 71-2122.

2 Professor, Department of Mathematics, University of Michigan, Ann Arbor, Michigan.

${ }^{3}$ Associate Professor, Department of Mathematics, Eastern Michigan University, Ypsilanti, Michigan. 
In Section 5, we shall state certain preliminary theorems which were proved by Cesari in Refs. 1, 2, and which we need in the proofs in Sections 6 and 8.

In Refs. 3, 4, Cesari has also proved other closure, lower closure, and semicontinuity theorems of a type different from those presented here, and also without seminormality conditions (or with very mild conditions of this kind). For the sake of brevity, we shall mention here only one semicontinuity theorem of this type in Remark 8 in Section 10.

\section{Notations}

Points in $E_{v}, E_{n}, E_{m}, E_{r+1}$ spaces will be denoted by $t=\left(t^{1}, \ldots, t^{v}\right)$, $x=\left(x^{1}, \ldots, x^{n}\right), u=\left(u^{1}, \ldots, u^{m}\right)$, and $\left(z^{0}, z\right)=\left(z^{0}, z^{1}, \ldots, z^{r}\right)$ or $(\eta, \xi)=$ $\left(\eta, \xi^{1}, \ldots, \xi^{r}\right)$, respectively. Also, $A$ is a given subset of the $t x$-space $E_{v} \times E_{n}, A_{0}$ is the projection of $A$ on $E_{v}$, and for $t \in A_{0}$ let $A(t)=$ $\left\{x \in E_{n} \mid(t, x) \in A\right\}$. For every $t \in A_{0}$ a subset $U(t)$ of $E_{m}$ is assigned, and $M$ denotes the set $\{(t, x, u) \mid(t, x) \in A, u \in U(t)\} \subset E_{v} \times E_{n} \times E_{m}$. Let $f_{0}(t, x, u)$ and $f(t, x, u)=\left(f_{1}, \ldots, f_{r}\right)$ be given functions on $M$.

We shall denote by $G$ and $T_{0}$ given subsets of $A_{0}$ in $E_{p}$, and $A_{G}$ and $H_{0}$ are the subsets $A_{G}=A \cap\left(G \times E_{n}\right), H_{0}=A \cap\left(T_{0} \times E_{n}\right)$. We shall denote by $|Z|$ the Lebesgue measure of a subset $Z$ of $E_{p}$.

We say that condition (C) is satisfied provided, given $\epsilon>0$, there is a compact subset $K$ of $G$ such that $|G-K|<\epsilon$, the set $A_{K}=$ $A \cap\left(K \times E_{n}\right)$ is closed, the set $M_{K}=\{(t, x, u) \mid t \in K\}$ is closed, and the functions $f_{0}(t, x, u), f(t, x, u)$ are continuous on $M_{K}$. In Mayer problems, we shall disregard $f_{0}$; in free problems, $f=u$. Condition (C) implies in particular that, for almost all $\bar{t} \in G$, the sets $A(\bar{t})$ and $M(\bar{t})=$ $\{(x, u) \mid(\vec{t}, x, u) \in M\}$ are closed, and $f_{0}(\vec{t}, x, u), f(\vec{t}, x, u)$ are continuous on $M(\bar{t})$.

Condition (C) is certainly satisfied if the sets $A$ and $M$ are closed and if $f_{0}$ and $f$ are measurable in $t$ and continuous in $(x, u)$ and $M(t)=$ $M\left(t^{\prime}\right)$ for $t, t^{\prime} \in G$. In particular, if $f_{0}$ and $f$ are continuous on $M$, and $A$ and $M$ are closed, then obviously condition (C) holds.

Whenever $f_{0}$ and $f$ have property (C) and $x(t), u(t), t \in G$, are measurable functions, then

$$
\begin{aligned}
& \eta(t)=f_{0}(t, x(t), u(t))=F_{0}[x, u](t), \quad t \in G, \\
& \xi(t)=f(t, x(t), u(t))=F[x, u](t), \quad t \in G,
\end{aligned}
$$

are measurable functions on $G$. Actually, (1) define operators $F_{0}, F$ from 
the space $(\mathscr{H}(G))^{n+m}$ of measurable functions $x(t)=\left(x^{1}, \ldots, x^{n}\right), u(t)=$ $\left(u^{1}, \ldots, u^{m}\right), t \in G$, into the spaces $\mathscr{M}(G)$ and $(\mathscr{M}(G))^{r}$ of the measurable functions $\eta_{0}(t), \xi(t)=\left(\xi^{1}, \ldots, \xi\right), t \in G$. These operators are often denoted as Carathéodory, or Nemytskii, operators (see, e.g., Ref. 5). Properties of these operators will be mentioned as needed.

\section{Property (D)}

With the notations as in the previous section, let $G$ be any measurable subset of $A_{0}$ with finite measure, $G \subset A_{0} \subset E_{v}$. Let $f$ be defined on $M$ and we shall assume that condition $(\mathrm{C})$ holds. We shall consider measurable functions $x(t)=\left(x^{1}, \ldots, x^{n}\right), u(t)=\left(u^{1}, \ldots, u^{m}\right), t \in G$. Under the sole Carathéodory-type continuity condition (C) on $f$, we know that the corresponding functions $\xi(t)=f(t, x(t), u(t)), t \in G$, also are measurable.

Given a sequence of measurable functions $x(t), x_{k}(t), u_{k}(t), t \in G$, $k=1,2, \ldots$, with $x(t), x_{k}(t) \in A(t), u_{k}(t) \in U(t), t \in G$ (a.e.), there are natural hypotheses (Section 5) under which the differences

$$
\delta_{k}(t)=f\left(t, x_{k}(t), u_{k}(t)\right)-f\left(t, x(t), u_{k}(t)\right), \quad t \in G, k=1,2, \ldots,
$$

belong to $\left(L_{1}(G)\right)^{r}$ and $\left\|\delta_{k}\right\|_{1} \rightarrow 0$ as $k \rightarrow \infty$. When this occurs, we say, for the sake of brevity, that property $(\mathscr{D})$ holds for the given sequence. The following general statement is of interest.

Statement 3.1. Let $f$ satisfy continuity condition (C) on $M$, and let $G$ be a measurable set of finite measure, $G \subset A_{0} \subset E_{y}$. Let $x(t)$, $x_{k}(t), u_{l}(t), t \in G, k=1,2, \ldots$, be a sequence of measurable a.e. finite functions, such that $x_{k}(t) \in A(t), u_{k}(t) \in U(t), t \in G$ (a.e.), $u_{k} \in\left(L_{1}(G)^{m}\right.$, $\left\|u_{t_{0}}\right\|_{1} \leqslant M_{0}$, a given constant, and $x_{k}(t) \rightarrow x(t)$ in measure in $G$ as $k \rightarrow \infty$, and the differences $\delta_{k}(t)=f\left(t, x_{k}(t), u_{k}(t)\right)-f\left(t, x(t), u_{k}(t)\right)$, $t \in G, k=1,2, \ldots$, are of class $\left(L_{1}(G)\right)^{r}$. Then $\delta_{k} \rightarrow 0$ strongly in $\left(L_{1}(G)\right)^{r}$ iff the same functions $\delta_{k}(t), t \in G, k=1,2, \ldots$, are equiabsolutely integrable in $G$.

Proof. Necessity. If $\delta_{k t} \rightarrow 0$ strongly in $\left(L_{1}(G)\right)^{r}$, then $\int_{G}\left|\delta_{k}(t)\right| d t \rightarrow 0$ as $k \rightarrow \infty$, and obviously the functions $\delta_{k}$ are equiabsolutely integrable.

Sufficiency. Let us assume that the functions $\delta_{k}$ are equiabsolutely integrable in $G$. Then, given $\epsilon>0$, there is $\sigma>0$ such that $\int_{H}\left|\delta_{k}(t)\right| d t \leqslant \epsilon$ for all $k=1,2, \ldots$, and any measurable subset $H$ of $G$ with $|H| \leqslant \sigma$. By property (C), there is a compact subset $K$ of $G$ with $|G-K|<\sigma / 4$, such that the set $M_{k}=[(t, x, u) \in M, t \in K]$ is closed 
and $f(t, x, u)$ is continuous on $M_{K}$. Since $x$ is measurable (and finite a.e. in $G)$, there is an integer $\lambda>0$ such that the set $A=[t \in G|| x(t) \mid \leqslant \lambda]$ has measure $\geqslant|G|-\sigma \mid 8$. Now the set $M^{\prime}$ of all $(t, x, u) \in M$ with $t \in K,|x| \leqslant \lambda+1,|u| \leqslant 4 M_{0} / \sigma$ is certainly compact, and $f$ is uniformly continuous on $M^{\prime}$. Hence, there is $\eta, 0<\eta \leqslant 1$, such that $(t, x, u)$, $(t, y, u) \in M^{\prime},|x-y| \leqslant \eta$ implies $|f(t, x, u)-f(t, y, u)|<\epsilon /|G|$. If $N=4 M_{0} \mid \sigma$, for any $k$ the set $P_{k}$ of all $t \in G$ with $\left|u_{k}(t)\right| \geqslant N$ has measure $\left|P_{k}\right|$ satisfying $N\left|P_{k}\right| \leqslant M_{0}$, hence, $\left|P_{k}\right| \leqslant M_{0} \mid N=\sigma / 4$. Finally, since $x_{k} \rightarrow x$ in measure, there is some integer $k_{0}$ such that for $k \geqslant k_{0}$ the set $\Lambda_{k}=\left[t \in G|| x_{k}(t)-x(t) \mid \leqslant \eta\right]$ has measure $\left|\Lambda_{k}\right|>$ $|G|-\sigma / 4$. Then, the set $A_{k}{ }^{\prime}=A_{k} \cap\left(G-P_{k}\right) \cap K \cap A$ has measure $\geqslant|G|-\sigma$, or $\left|G-\Lambda_{k}^{\prime}\right| \leqslant \sigma$. We have now $\int_{G-A_{k}^{\prime}}\left|\delta_{k}(t)\right| d t \leqslant \epsilon$. On the other hand, for $t \in \Lambda_{k_{i}}{ }^{\prime}$ we have $t \in K,\left|x_{k}(t)-x(t)\right| \leqslant \eta \leqslant 1$, $|x(t)| \leqslant \lambda, \quad\left|x_{k_{k}}(t)\right| \leqslant \lambda+1, \quad\left|u_{k}(t)\right| \leqslant N=4 M_{0} / \sigma, \quad\left(t, x(t), \quad u_{k}(t)\right)$, $\left(t, x_{k}(t), u_{k}(t)\right) \in M^{\prime}$, and $\left|f\left(t, x(t), u_{k}(t)\right)-f\left(t, x_{k}(t), u_{k}(t)\right)\right| \leqslant \epsilon|| G \mid$ and this holds for all $t \in K, k \geqslant k_{0}$, with $|G-K|<\sigma / 4$. We have proved that $\delta_{k} \rightarrow 0$ in measure. On the other hand $\int_{\Lambda_{k}^{\prime}}\left|\delta_{k}(t)\right| d t \leqslant \epsilon$, and finally $\int_{G}\left|\delta_{k}(t)\right| d t \leqslant 2 \epsilon$ for all $k \geqslant k_{0}$. Thus, $\left\|\delta_{k}\right\|_{1} \rightarrow 0$ as $k \rightarrow \infty$. Statement 3.1 is thereby proved.

The same Statement 3.1 above holds, naturally, for the differences

$$
\delta_{k}^{0}(t)=f_{0}\left(t, x_{k}(t), u_{k}(t)\right)-f_{0}\left(t, x_{k}(t), u_{k}(t)\right), \quad t \in G, \quad k=1,2, \ldots
$$

For the sake of brevity, we refer only to the differences $\delta_{l k}$.

Remark 3.1. The sufficiency part in Statement 3.1 can also be stated as follows. Let $f$ satisfy property (C). Let $S=\{x(t), u(t), t \in G\}$ denote a class of measurable functions $x, u$ in $G$ with the properties: (i) $x(t) \in A(t), u(t) \in U(t), t \in G$; (ii) for every $\sigma>0$ there are constants $M_{1}(\sigma), M_{2}(\sigma)>0$, such that the sets $\left[t \in G|| x(t) \mid \leqslant M_{1}(\sigma)\right]$, $\left[t \in G|| u(t) \mid \leqslant M_{2}(\sigma)\right]$ have measures $\geqslant|G|-\sigma$; (iii) for any two pairs $(x, u),(y, u)$ in $S$ the differences $\delta(t)=f(t, x(t), u(t))-f(t, y(t)$, $u(t)), t \in G$, belong to $\left(L_{1}(G)\right)^{r}$ and are equiabsolutely integrable in $G$. Then $\|\delta\|_{1} \rightarrow 0$ uniformly in $S$ as $x-y \rightarrow 0$ in measure. The proof is the same as for Statement 3.1. If condition (iii) is not satisfied, then $\delta(t) \rightarrow 0$ in measure uniformly in $S$ as $x-y \rightarrow 0$ in measure. The equiabsolute integrability of $\delta(t)$ in (iii) is guaranteed by the inequalities on $\delta$ in the conditions $(\mathrm{F}),(\mathrm{G})$, and $(\mathrm{H})$ below. Finally, we note also that condition (ii) above is certainly satisfied if $x \in\left(L_{1}(G)\right)^{n},\|x\|_{1} \leqslant L_{0}$, and there is a continuous function $\phi(u), \phi: E^{m} \rightarrow E^{m}$, with $|\phi(u)| \rightarrow \infty$ as $|u| \rightarrow \infty$ such that $\phi \circ u \in\left(L_{1}(G)\right)^{m}$ and $\|\phi \circ u\|_{1} \leqslant L, L_{0}, L$ given constants. 


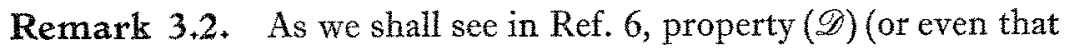
$\delta_{k} \rightarrow 0$ in measure) implies a weak form of seminormality [a property of the (Q)-type, according to the terminology in Refs. 1 and 5]. Such form of property $(\mathrm{Q})$ is not so weak, after all, since it could be used to reprove closure and lower closure theorems by the usual approach (Ref. 1).

Remark 3.3. The reader should compare Statement 3.1 above with the statement in Ref. 7, page 122, No. 6, that, for $|G|<\infty$, and given $L_{1}$-functions $\delta_{k}$ defined on $G$, then $\left\|\delta_{k}\right\|_{1} \rightarrow 0$ iff $\delta_{k} \rightarrow 0$ in measure, and the same functions are equiabsolutely integrable. In the proof of Statement 3.1 above, we have indeed shown that $\delta_{k} \rightarrow 0$ in measure. Once this was done, the rest of the proof was only one line. The reader should also compare our proof of the convergence $\delta_{k} \rightarrow 0$ in measure with the statement in Ref. 5 , page $20,(2.1)$, that a Carathéodory operator $\mathscr{F}$, acting from a space $L_{p}$ into a space $L_{q}, 1 \leqslant p, q<+\infty$, transforms every sequence $u_{k} \in L_{p}$ convergent in measure into a sequence $v_{k}=$ $\mathscr{F} u_{k} \in L_{q}$ also convergent in measure.

Note Added in Proof. The reader should compare the proof of Statement 3.1 with the proof of Theorem 17.4 on p. 355 of Ref. 14. Statement 3.1, however, does not follow from this theorem or those quoted in Remark 3.3 .

\section{Criteria for Property (D)}

We shall now discuss alternate hypotheses, each of which guarantees that $\delta_{k} \in\left(L_{1}(G)\right)^{r}$ and $\delta_{k} \rightarrow 0$ strongly in $\left(L_{1}(G)\right)^{r}$ as $k \rightarrow \infty$. We shall always assume below that $f$ satisfies Carathéodory-type continuity condition (C).

Note that, under the conditions (F) below, we assume that $x_{k} \in\left(L_{p}(G)\right)^{n}$ but the $u_{k}$ are only measurable; under the conditions $(\mathrm{G})$, we assume that $x_{k} \in\left(L_{p}(G)\right)^{n}$, $\phi \circ u_{k} \in\left(L_{q}(G)\right)^{m}$; under the conditions (H), we assume that $u_{k} \in\left(L_{q}(G)\right)^{m}$ but the $x_{k}$ are only measurable.

\section{A. Lipschitz-Type Conditions (F)}

$\left(\mathrm{F}_{p}\right)$ (i) For $1 \leqslant p<\infty, x, x_{k} \in\left(L_{p}(G)\right)^{n},\left\|x_{k}-x\right\|_{p} \rightarrow 0$, and (ii) $\left|f\left(t, x_{k}(t), u_{k}(t)\right)-f\left(t, x(t), u_{k}(t)\right)\right| \leqslant F_{l}(t) h\left(\left|x_{k}(t)-x(t)\right|\right), t \in G$, $k=1,2, \ldots$, where $h(\zeta), 0 \leqslant \zeta<+\infty$, is a given monotone nondecreasing function with $h(0+)=0, h(\zeta) \leqslant c|\zeta| \gamma, c \geqslant 0,0<\gamma \leqslant p$, for all $\zeta \geqslant \zeta_{0} \geqslant 0\left(c, \gamma, \zeta_{0}\right.$ given constants $)$, and $F_{k}(t) \geqslant 0, t \in G, F_{k} \in L_{p^{\prime}}(G)$, 
given functions with $p^{\prime}=p /(p-\gamma),\left(p^{\prime}=\infty\right.$ if $\left.\gamma=p\right)$, and $\left\|F_{k}\right\|_{p^{\prime}} \leqslant C$, a given constant.

Let us prove that conditions (C) and $\left(\mathrm{F}_{p}\right)$ imply $\left\|\delta_{k}\right\|_{1} \rightarrow 0$ as $k \rightarrow \infty$. Given $\epsilon>0$, let $\sigma>0$ be so chosen that $C \sigma^{\gamma / p} h\left(\zeta_{0}\right) \leqslant \epsilon$. It is to be noted that, since $\gamma>0$, we have $p^{\prime}>1$. If $p^{\prime}<\infty$ let $p^{\prime \prime}$ be defined by $1 / p^{\prime}+1 / p^{\prime \prime}=1$, so that $p^{\prime \prime}=p / \gamma$ and then, by Hölder inequality, we have for any measurable subset $A$ of $G, \int_{A}\left|F_{k}(t)\right| \leqslant|A|^{\gamma / p} C$, where $|A|$ is the measure of $A$. In particular, $\left\|\boldsymbol{F}_{k}\right\|_{1} \leqslant C^{\prime}$ where $C^{\prime}=$ $|G|^{\gamma / p} C$. If $p^{\prime}=\infty$, then $C^{\prime}=|G| \cdot C$. Let $\eta>0$ be now so chosen that $h(\eta) C^{\prime} \leqslant \epsilon$. Now, if $\left\|x_{k}-x\right\|_{p} \rightarrow 0$, then $\left\|x_{k}-x\right\|_{1} \rightarrow 0$ and also $x_{k}-x \rightarrow 0$ in measure. Thus, there is some $k_{0}$ such that, for all $k \geqslant k_{0}$, we have $\left\|x_{k}-x\right\|_{p}<(\epsilon / C c)^{1 / \gamma}$ and the set $G_{1 k}=\left[t \in G|| x_{k}(t)-x(t) \mid \leqslant \eta\right]$ has measure $>|G|-\sigma$. We consider also the sets $G_{2 k}=[t \in G \mid \eta<$ $\left.\left|x_{k}(t)-x(t)\right|<\zeta_{0}\right]$ and $G_{3 k}=\left[t \in G|| x_{k}(t)-x(t) \mid \geqslant \zeta_{0}\right]$. Then $\left|G_{2 k}\right|<\sigma,\left|G_{3 k}\right|<\sigma$; and, by Hölder inequality and the definitions above, we have for $k \geqslant k_{0}$

$$
\begin{aligned}
\int_{G}\left|\delta_{k}(t)\right| d t & =\left(\int_{G_{1 k}}+\int_{G_{2 k}}+\int_{G_{2 k}}\right)\left|\delta_{k}(t)\right| d t \\
& \leqslant h(\eta) \int_{G} F_{k}(t) d t+\left|G_{2 z}\right|^{\nu / p} C h\left(\zeta_{0}\right)+c \int_{G} F_{k}\left|x_{k}-x\right|^{\gamma} d t \\
& \leqslant C^{\prime} h(\eta)+\sigma^{\gamma / p} C h\left(\zeta_{0}\right)+c C\left(\left\|x_{k}-x\right\|_{p}\right)^{\gamma} \leqslant 3 \epsilon_{0}
\end{aligned}
$$

We have proved that, if $\left\|x_{k}-x\right\|_{p} \rightarrow 0$, then $\left\|\delta_{k}\right\|_{1} \rightarrow 0$ under the hypotheses $(C)$ and $\left(F_{p}\right)$.

It is to be noted that the above proof uses the fact that the functions $F_{k}, k=1,2, \ldots$, are equiabsolutely integrable under the stated conditions, in particular $\gamma>0$. If $\gamma=0$, that is, $p^{\prime}=1$, then we need to assume that $F_{k}$ are equiabsolutely integrable and it is not enough to assume $\left\|F_{k}\right\|_{1} \leqslant C$. However, in this case, $\delta_{k}$ automatically are equiabsolutely integrable (because of the condition on $f$ ) and, by Statement 3.1, $\left\|\delta_{k}\right\|_{1} \rightarrow 0$.

Remark 4.1. The part concerning $f$ in $\left(F_{p}\right)$ above could be stated in the form

$$
|f(t, x, u)-f(t, y, u)| \leqslant F(t, u) h(|x-y|)
$$

for all $(t, x, u),(t, y, u) \in M$, where $h(\zeta), 0 \leqslant \zeta<+\infty$, is as stated in $\left(\mathrm{F}_{p}\right)$ and $F(t, u) \geqslant 0$ is a function defined, say, on $G \times E_{m}$. Then for the class of controls $u(t), t \in G$, to be taken into consideration we may require that $F(t, u(t)) \in L_{p^{\prime}}(G),\|F(t, u(t))\|_{p^{\prime}} \leqslant C$. An analogous remark holds also for other conditions below but we shall not repeat it for the sake of brevity. 
Note that property $\left(\mathrm{F}_{p}\right)$ does not guarantee that the single functions $f\left(t, x(t), u_{k}(t)\right), f\left(t, x_{k}(t), u_{k}(t)\right), t \in G, k=1,2, \ldots$, are of class $\left(L_{1}(G)\right)^{r}$ [only the differences $\delta_{k} \in\left(L_{1}(G)\right)^{r}$ ]. We may add to $\left(\mathrm{F}_{p}\right)$ the requirement below.

$\left(\mathrm{F}_{p}{ }^{\prime}\right)$ There is a function $\psi(t) \geqslant 0, t \in G, \psi \in L_{\mathrm{I}}(G)$, and a constant $c \geqslant 0$ such that, for all $(t, x) \in A_{G}$ and $u \in U(t)$, we have

$$
|f(t, x, u)| \leqslant \psi(t)+c|x|^{p} .
$$

We shall denote by $\left(\mathrm{F}_{p}{ }^{*}\right)$ the union of condition $\left(\mathrm{F}_{p}\right)$ above (in any form), and of $\left(\mathrm{F}_{p}{ }^{\prime}\right)$. Under condition $\left(\mathrm{F}_{p}{ }^{*}\right)$, then certainly the single functions $f\left(t, x(t), x_{k}(t)\right), f\left(t, x_{k}(t), u_{k}(t)\right)$ are of class $\left(L_{1}(G)\right)^{r}$.

$\left(\mathrm{F}_{\infty}\right) x, x_{k} \in L_{\infty}(G),\left\|x_{k}-x\right\|_{\infty} \rightarrow 0$, and

$$
\left|f\left(t, x_{k k}(t), u_{k}(t)\right)-f\left(t, x(t), u_{k}(t)\right)\right| \leqslant F_{k}(t) h\left(\left|x_{k}(t)-x(t)\right|\right), \quad t \in G,
$$

$k=1,2, \ldots$, where $h(\zeta) \geqslant 0,0 \leqslant \zeta<+\infty$, is a given monotone nondecreasing functions with $h(0+)=0$, and $F_{k}(t) \geqslant 0, t \in G, F_{k} \in L_{1}(G)$, are given function with $\left\|F_{k}\right\|_{i} \leqslant C$, a given constant.

Let us prove that conditions (C) and $\left(\mathrm{F}_{\infty}\right)$ imply $\left\|\delta_{k}\right\|_{1} \rightarrow 0$. Let $\epsilon>0$ be given, let us choose $\eta>0$ so that $C h(\eta)<\epsilon$, and let us choose $k_{0}$ so that $k \geqslant k_{0}$ implies $\left\|x_{k}-x\right\|_{\infty}<\eta$; hence, $\left|x_{k}(t)-x(t)\right| \leqslant$ $\left\|x_{k}-x\right\|_{\infty} \in \eta$ a.e. in $G$. For $k \geqslant k_{0}$ we have then

$$
\int_{G}\left|\delta_{t}\right| d t \leqslant \int_{G} F_{l c}(t) h\left(\left\|x_{k}-x\right\|_{\infty}\right) d t \leqslant C h(\eta)<\epsilon .
$$

As above, we may also denote by $\left(\mathrm{F}_{\infty}{ }^{*}\right)$ the union of $\left(\mathrm{F}_{p}\right)$ and of the following further requirement, analogous to $\left(\mathrm{F}_{p}{ }^{\prime}\right)$.

$\left(F_{\infty}{ }^{\prime}\right)$ There are a function $\psi(t) \geqslant 0, t \in G, \psi \in L_{1}(G)$, and a monotone nondecreasing function $\sigma(\zeta) \geqslant 0,0 \leqslant \zeta<+\infty$, such that, for all $(t, x, u) \in M$, we have

$$
|f(t, x, u)| \leqslant \psi(t) o(|x|) .
$$

Note that, for $f$ linear in $x$, that is, of the form $f(t, x, u)=B(t, u) x+$ $C(t, u), B=\left[b_{i j}(t, u)\right], C=\left[c_{i}(t, u)\right]$ matrices of the types $r \times n, r \times 1$, with $\left|b_{i j}(t, u)\right| \leqslant \Phi(t)$, for $\Phi(t) \geqslant 0, t \in G, \Phi \in L_{p^{\prime}}(G)$, as in $\left(\mathrm{F}_{p}\right)$ with $\gamma=1,1 \leqslant p<\infty$, then condition $\left(\mathrm{F}_{p}\right)$ is certainly satisfied. For $p=\infty$, $\Phi \in L_{1}(G)$, then condition ( $\left.\mathrm{F}_{\infty}\right)$ is satisfied.

\section{B. Growth-Type Conditions (G)}

$\left(\mathrm{G}_{p q}\right)$ (i) There exists a continuous function $\phi(u), \phi: E^{m} \rightarrow E^{m}$, with $|\phi(u)| \rightarrow \infty$ as $|u| \rightarrow \infty$, such that for $1 \leqslant p, q<\infty, x, x_{k} \in\left(L_{p}(G)\right)^{n}$, 
$u_{k}$ measurable, $\phi \circ u_{k} \in\left(L_{q}(G)\right)^{m},\|x\|_{p},\left\|x_{k}\right\|_{p} \leqslant L_{0},\left\|\phi\left(u_{k}\right)\right\|_{q} \leqslant L$, ( $L_{0}, L$ given constants), $x_{k}(t) \rightarrow x(t)$ in measure in $G$ as $k \rightarrow \infty$, and (ii) there are constants $c, c^{\prime}, \alpha, \beta$ with $c, c^{\prime} \geqslant 0,0<\alpha \leqslant p, 0<\beta \leqslant q$, and a function $\psi(t) \geqslant 0, t \in G, \psi \in L_{1}(G)$, such that, for all $(t, x, u)$, $(t, y, u) \in M$, we have

$$
|f(t, x, u)-f(t, y, u)| \leqslant \psi(t)+c\left(|x|^{p-\alpha}+|y|^{p-\alpha}\right)+c^{\prime}|\phi(u)|^{q-\beta} .
$$

Let us prove that conditions (C) and $\left(\mathrm{G}_{p q}\right)$ imply $\left\|\delta_{l i}\right\|_{1} \rightarrow 0$. Indeed, let $p^{\prime \prime}, q^{\prime \prime}$ be defined by $1 / p^{\prime \prime}+(p-\alpha) / p=1,1 / q^{\prime \prime}+(q-\beta) / q=1$, so that $p^{\prime \prime}=p / \alpha, q^{\prime \prime}=q / \beta$. For any measurable subset $H$ of $G$ we have, by Hölder inequality,

$$
\begin{aligned}
\int_{H}\left|\delta_{k}\right| d t & \leqslant \int_{H} \psi(t) d t+c \int_{H}\left(\left|x_{k}(t)\right|^{p-\alpha}+|x(t)|^{p-\alpha}\right) d t+c^{\prime} \int_{H}\left|\phi\left(u_{k}(t)\right)\right|^{q-\beta} d t \\
& \leqslant \int_{H} \psi(t) d t+c|H|^{\alpha / p}\left(\left\|x_{k}\right\|_{p}^{p-\alpha}+\|x\|_{p}^{p-\alpha}\right)+c^{\prime}|H|^{\beta / q}\left\|\phi\left(u_{k}(t)\right)\right\|_{q}^{q-\beta} \\
& \leqslant \int_{H} \psi(t) d t+2 c L_{0}^{p-\alpha}|H|^{\alpha / p}+c^{\prime} L^{\alpha-\beta}|H|^{\beta / q} .
\end{aligned}
$$

The last member obviously approaches zero as $|H| \rightarrow 0$, and the equiabsolute integrability of the functions $\delta_{k}(t), t \in G$, is proved. Then $\left\|\delta_{k}\right\|_{1} \rightarrow 0$ by force of Statement 3.1 and Remark 3.1.

Note that the inequality $\left(\mathrm{G}_{p q}\right)$ is certainly satisfied if we know that $p>L$ and that $|f(t, x, u)-f(t, y, u)| \leqslant \psi(t)+c|x-y|+c^{\prime}|u|^{q-\beta}$.

Remark 4.2. The following statement is of interest. Let $S=$ $\{x(t), u(t), t \in G\}$ denote any class of functions with $x(t) \in A(t), u(t) \in U(t)$, $t \in G$, a.e., $x \in\left(L_{p}(G)\right)^{m}, u \in\left(L_{q}(G)\right)^{m},\|x\|_{p} \leqslant L_{0},\|u\|_{q} \leqslant L,\left(L_{0}, L\right.$ given constants), and let $f$ satisfy conditions $(C)$ and $\left(\mathrm{G}_{p q}\right)$ above. For any two pairs $(x, u),(y, u)$ in $S$ let us consider the differences $\delta(t)=$ $f(t, x(t), u(t))-f(t, y(t), u(t)), t \in G$. Then $\delta \in\left(L_{1}(G)\right)^{r}$ and, the same $\delta(t), t \in G$, are equiabsolutely integrable, and $\|\delta\|_{1} \rightarrow 0$ uniformly in $S$ as $x(t)-y(t) \rightarrow 0$ in measure. The proof is the same as above where Remark 3.1 is used.

$\left(\mathrm{G}_{\infty q}\right)$ (i) There exists a continuous function $\phi(u), \phi: E^{m} \rightarrow E^{m}$, with $|\phi(u)| \rightarrow \infty$ as $|u| \rightarrow \infty$, such that for $1 \leqslant q<\infty, x, x_{k} \in\left(L_{\infty}(G)\right)^{n}$, $\phi\left(u_{k}\right) \in\left(L_{q}(G)\right)^{m},\|x\|_{\infty},\left\|x_{k}\right\|_{\infty} \leqslant L_{0},\left\|\phi\left(u_{k}\right)\right\|_{q} \leqslant L, \quad\left(L, L_{0}\right.$ given constants), $x_{k}(t) \rightarrow x(t)$ in measure in $G$ as $k \rightarrow \infty$, and (ii) there are constants $c^{\prime}, \beta, 0<\beta \leqslant q$, a function $\psi(t) \geqslant 0, t \in G, \psi \in L_{1}(G)$, and a monotone nondecreasing function $\sigma(\zeta) \geqslant 0,0 \leqslant \zeta<+\infty$, such that for all $(t, x, u),(t, y, u) \in M$ we have

$$
|f(t, x, u)-f(t, y, u)| \leqslant \psi(t) \sigma(|x|+|y|)+c^{\prime}|\phi(u)|^{\alpha-\beta} .
$$


Let us prove that conditions $(C)$ and $\left(G_{\infty \propto q}\right)$ imply $\left\|\delta_{k}\right\|_{1} \rightarrow 0$. Indeed, note that the requirements $\left\|x_{k}\right\|_{\infty},\|x\|_{\infty} \leqslant L_{0}$ imply $\mid x_{k}(t)$, $|x(t)| \leqslant L_{0}, t \in G$, (a.e.). From this and $\left\|\phi\left(u_{k}\right)\right\|_{q} \leqslant L$, we derive as above, by Hölder inequality, that

$$
\int_{H}\left|\delta_{k_{i}}(t)\right| d t \leqslant \sigma\left(2 L_{0}\right) \int_{H} \psi(t) d t+c L^{q-\beta}|H|^{\beta / q} .
$$

Here $\psi$ is a fixed $L$-integrable function on $G$, hence absolutely integrable, and the last relation proves, therefore, that the functions $\delta_{k}(t), t \in G$, $k=1,2, \ldots$, are equiabsolutely integrable. Then $\left\|\delta_{k}\right\|_{1} \rightarrow 0$ by force of Statement 3.1.

Here too inequality $\left(G_{\infty q}\right)$ is certainly satisfied if we know that

$$
|f(t, x, u)-f(t, y, u)| \leqslant \psi(t) \sigma(|x-y|)+c^{\prime}|\phi(u)|^{q-\beta} .
$$

Note that, in $\left(\mathrm{G}_{p q}\right),\left(\mathrm{G}_{\infty q}\right)$, we do not assume $\sigma(0+)=0$. These conditions $\left(\mathrm{G}_{p q}\right),\left(\mathrm{G}_{\infty q}\right)$ are only growth conditions, the continuity property of $f$ (property $C$ ) having the main role in the proof that $\delta_{k} \rightarrow 0$ strongly in $\left(L_{1}(G)\right)^{r}$.

We leave to the reader to state conditions $\left(G_{p \infty}\right)$ and $\left(G_{\infty \infty}\right)$ analogous to the ones above.

Note that none of the properties $\left(\mathrm{G}_{p q}\right)$ above, $1 \leqslant p, q \leqslant \infty$, can guarantee that the single functions $f\left(t, x(t), u_{k}(t)\right), f\left(t, x_{k}(t), u_{k}(t)\right), t \in G$, $k=1,2, \ldots$, is of class $\left(L_{p}(G)\right)^{r}$ (but the differences $\delta_{k}$ are in this class). When needed, we may denote by $\left(\mathrm{G}_{p q}^{*}\right), 1 \leqslant p, q \leqslant \infty$, the union of $\left(\mathrm{G}_{p q}\right)$ and of the further requirement that, for all $(t, x, u) \in M$, we have

$$
\begin{aligned}
& \left(\mathrm{G}_{p a}^{\prime}\right) \quad|f(t, x, u)| \leqslant \psi(t)+c|x|^{p}+c^{\prime}|\phi(u)|^{\alpha} \quad \text { if } \quad 1 \leqslant p, q<\infty, \\
& \left(\mathrm{G}_{\infty \infty}^{\prime}\right)|f(t, x, u)| \leqslant \psi(t) \sigma(|x|)+c^{\prime}|\phi(u)|^{\alpha} \quad \text { if } \quad p=\infty, 1 \leqslant q<\infty,
\end{aligned}
$$

and analogous requirements $\left(G_{p \infty}^{\prime}\right),\left(G_{\infty \infty}^{\prime}\right)$.

Note that, if relation $\left(\mathrm{G}_{p q}\right)$ holds as stated, and relation $\left(\mathrm{G}_{p q}^{\prime}\right)$ holds for all $u$ and a fixed $x(t), t \in G, x \in\left(L_{p}(G)\right)^{n}$, then $\left(\mathrm{G}_{p q}^{\prime}\right)$ holds for all $(t, x, u) \in M$ as stated. Indeed,

$$
\begin{aligned}
|f(t, x, u)| \leqslant & |f(t, x(t), u)|+|f(t, x, u)-f(t, x(t), u)| \\
\leqslant & {\left[2 \psi(t)+c|x(t)|^{p}+c|x(t)|^{p-\alpha}+c+c^{\prime}\right] } \\
& +c^{\prime}|\phi(u)|^{\alpha}+c\left(|x|^{p-\alpha}-1\right)+c^{\prime}\left(|\phi(u)|^{q-\beta}-1\right) \\
\leqslant & \psi_{0}(t)+c|x|^{p}+2 c^{t}|\phi(u)|^{q} .
\end{aligned}
$$

An analogous remark holds for the other conditions. 


\section{Growth-Type Conditions (H)}

$\left(\mathrm{H}_{q}\right)$ (i) For $1 \leqslant q<\infty, x, x_{k}$ measurable, $x_{k}(t) \rightarrow x(t)$ in measure in $G$ as $k \rightarrow \infty, u_{k} \in\left(L_{q}(G)\right)^{m},\left\|u_{k}\right\|_{q} \leqslant L$, a constant, and (ii) there are other constants $c^{\prime}, \beta, 0<\beta \leqslant q$, and a function $\psi(t) \geqslant 0, t \in G, \psi \in L_{1}(G)$, such that, for all $(t, x, u),(t, y, u) \in M$, we have

$$
|f(t, x, u)-f(t, y, u)| \leqslant \psi(t)+c^{\prime}|u|^{\alpha-\beta} .
$$

The proof that conditions (C) and $\left(\mathrm{H}_{q}\right)$ imply $\left\|\delta_{k}\right\|_{1} \rightarrow 0$ is the same as for $\left(\mathrm{G}_{\infty q}\right)$.

$\left(\mathrm{H}_{\infty}\right)$ (i) $x, x_{k}$ measurable, $x_{k}(t) \rightarrow x(t)$ in measure in $G$ as $k \rightarrow \infty$, $u_{k} \in\left(L_{\infty}(G)\right)^{m},\left\|u_{k}\right\|_{\infty} \leqslant L$, and (ii) there are a function $\psi(t) \geqslant 0$, $t \in G, \psi \in L_{1}(G)$, and a monotone nondecreasing function $\sigma(\zeta) \geqslant 0$, $0 \leqslant \zeta<+\infty$, such that, for all $(t, x, u),(t, y, u) \in M$, we have $|f(t, x, u)-f(t, y, u)| \leqslant \psi(t) \sigma(|u|)$.

When needed, we shall denote by $\left(\mathrm{H}_{q}{ }^{*}\right)$ the union of $\left(\mathrm{H}_{q}\right)$ and of the further requirement that, for all $(t, x, u) \in M$, we have

$$
|f(t, x, u)| \leqslant \psi(t)+c^{\prime}|u|^{q} .
$$

Example 4.1. Take $v=1, n=1, m=1, p=2, q=2, G=$ $[-a, a], x \in E_{1}, u \in E_{1}$. Then the function $f(t, x, u)=t+x+u^{2}$ satisfies condition $\left(\mathrm{G}_{22}\right)$, and so does the function $f(t, x, u)=t+$ $\sin (t x)+(1+\cos t x) x+(\sin t) u^{2}+(\sin x) u$ with $\alpha=\beta=1$.

Example 4.2. Take $v=1, n=1, m=1, q=2, G=[-a, a]$, $A=G \times E_{1}, U=E_{1}$. Then the function $f(t, x, u)=\exp (x)+u+u t^{2}$ certainly satisfies condition $\left(G_{\infty 2}\right)$, and so does the function $f(t, x, u)=$ $t^{-1 / 2} \exp \left(t x^{2}\right)+1+x(\cos t x)+(\sin x) u+(\sin t) u^{2}$, with $\psi(t)=t^{-1 / 2}$, $\sigma(\zeta)=\exp \left(a \zeta^{2}\right)+a^{1 / 2} \zeta$.

Example 4.3. Take $\nu=1, n=1, m=1, p=2, G=[-a, a]$, $A=G \times E_{1}, U=E_{1}$. Then, $f(t, x, u)=(\sin t+\arctan u) x+\exp (u)$ satisfies condition $\left(\mathrm{F}_{2}\right)$ with $\xi_{0}=1, \gamma=1, p^{\prime}=2$, and so does the function $f(t, x, u)=\left(t^{-2 / 5}+t \arctan u\right)\left(x+|x|^{1 / 2}\right)+\exp (u)$. The function $f(t, x, u)=\left(t^{-2 / 3}+t \arctan u\right)\left(x+|x|^{1 / 2}+|x|^{1 / 3}\right)+\exp (u)$ satisfies $\left(\mathrm{F}_{2}\right)$ with $\gamma=1, p^{\prime}=2$.

Example 4.4. Take $v=1, n=1, m=1, G=[-a, a], A=$ $G \times E_{1}, U=E_{1}$. Then, $f(t, x, u)=(\arctan x) u^{3 / 2}+u^{2}$ satisfies $\left(\mathrm{H}_{2}\right)$ with $\beta=1 / 2, \psi(t)=4$. The function $f(t, x, u)=\left(t^{-2 / 3}+\arctan x\right)$ $\exp (u)+\exp \left(u^{2}\right)$ satisfies $\left(\mathrm{H}_{\infty}\right)$ with $\sigma(\zeta)=\exp (\zeta), \psi(t)=4$. 
For $f(t, x, u)$ linear in $u$, that is, of the form

$$
f(t, x, u)=B(t, x) u+C(t, x)
$$

$B=\left[b_{i j}(t, x)\right], C=\left[c_{i}(t, x)\right]$ matrices of the types $r \times m, r \times 1$, suitable conditions can be stated in terms of the matrices $B$ and $C$. For instance:

$\left(\mathrm{GL}_{p q}\right)$ (i) For $1 \leqslant p<\infty, 1<q<\infty, x, x_{k} \in\left(L_{p}(G)\right)^{n}$, $u_{k} \in\left(L_{q}(G)\right)^{m},\|x\|_{p},\left\|x_{k}\right\|_{p} \leqslant L_{0},\left\|u_{h}\right\|_{q} \leqslant L, x_{l}(t) \rightarrow x(t)$ in measure, and (ii) there are constants $c, \alpha, 0<\alpha \leqslant p$, and a function $\psi(t), t \in G$, $\psi \in L_{1}(G)$, such that, for all $(t, x),(t, y) \in A_{G}$, we have

$$
\left|b_{i j}(t, x)\right| \leqslant c, \quad\left|c_{i}(t, x)-c_{i}(t, y)\right| \leqslant \psi(t)+c\left(|x|^{p-\alpha}+|y|^{p-\alpha}\right) .
$$

We need only to verify that $f$ satisfies condition $\left(G_{p q}\right)$. We have

$$
\begin{aligned}
|f(t, x, u)-f(t, y, u)| \leqslant & \sum_{i} \sum_{j}\left|b_{i j}(t, x)-b_{i j}(t, y)\right|\left|u^{j}\right| \\
& +\sum_{i}\left|c_{i}(t, x)-c_{i}(t, y)\right| \\
\leqslant & 2 m m c|u|+2 r \psi(t)+r c\left(|x|^{p-\alpha}+|y|^{p-\alpha}\right),
\end{aligned}
$$

and $\left(\mathrm{G}_{p q}\right)$ is satisfied with $\phi(u)=u, \beta=q-1$, and $\alpha$ as given.

Note that, under condition ( $\left(\mathrm{GL}_{p q}\right)$, the functions $B\left(t, x_{k}(t)\right) u_{k}(t)$, $t \in G, k=1,2, \ldots$, are of class $\left(L_{q}(G)\right)^{r}$ and have $L_{q}$-norms $\leqslant r m c L$. Thus, they always possess a subsequence which is weakly convergent in $\left(L_{q}(G)\right)^{r}$, and then also in $\left(L_{1}(G)\right)^{r}$. If, in addition to $\left(\mathrm{GL}_{p q}\right)$, we know that $\left(\mathrm{GL}_{p q}^{\prime}\right)\left|c_{i}(t, x)\right| \leqslant \psi(t)+c|x|^{p}$, then certainly the functions $\xi_{k}(t)=f\left(t, x_{k}(t), u_{k}(t)\right), k=1,2, \ldots$, belong to $\left(L_{1}(G)\right)^{r}$. If we also know that the functions $c_{i}\left(t, x_{k}(t)\right), t \in G, k=1,2, \ldots$, are equiabsolutely integrable, then there is certainly a subsequence $\left[k_{s}\right]$ such that $\xi_{k_{s}}$ converges weakly in $\left(L_{1}(G)\right)^{r}$. Analogous remarks hold also under the conditions which we list below, but we shall omit them for the sake of brevity.

$\left(\mathrm{GL}_{p 1}\right)$ For $1 \leqslant p<\infty, q=1$, the same as $\left(\mathrm{GL}_{p q}\right)$ with $q=1$, where now $u_{k} \in\left(L_{1}(G)\right)^{m}$, and the sequence $u_{k}$ is known to be equiabsolutely integrable in $G$.

The latter requirement is certainly satisfied if the sequence $u_{k}$ is known to converge weakly in $\left(L_{1}(G)\right)^{m}$.

To prove that conditions (C) and $\left(G L_{p 1}\right)$ imply that $\left\|\delta_{k}\right\|_{1} \rightarrow 0$, note that

$$
\begin{aligned}
\left|\delta_{k}(t)\right| & =\left|f\left(t, x_{k}(t), u_{k}(t)\right)-f\left(t, x(t), u_{k}(t)\right)\right| \\
& \leqslant 2 r m c\left|u_{k}(t)\right|+2 r \psi(t)+x c\left(\left|x_{k}(t)\right|^{p-\alpha}+\left|y_{k}(t)\right|^{p-\alpha}\right),
\end{aligned}
$$


and the sequence $\delta_{k}(t), t \in G, k=1,2, \ldots$, is then equiabsolutely integrable in $G$. Finally, $\left\|\delta_{k}\right\|_{1} \rightarrow 0$ by force of Statement 3.1.

$\left(\mathrm{GL}_{\infty q}\right)$ (i) For $1<q<\infty$, the same as in $\left(\mathrm{GL}_{p q}\right)$ where now $x$, $x_{k} \in L_{\infty}(G), u_{k} \in\left(L_{q}(G)\right)^{m}, x_{k}(t) \rightarrow x(t)$ in measure, $\left\|u_{k}\right\|_{q} \leqslant L$, and (ii) there are a constant $c$, a function $\psi(t), t \in G, \psi \in L_{1}(G)$, and a monotone nondecreasing function $\sigma(\zeta) \geqslant 0,0 \leqslant \zeta<+\infty$, such that, for all $(t, x) \in A_{G}$, we have $\left|b_{i j}(t, x)\right| \leqslant c,\left|c_{i}(t, x)\right| \leqslant \psi(t) \sigma(|x|)$.

$\left(\mathrm{GL}_{\infty 1}\right)$ The same as $\left(\mathrm{GL}_{\infty q q}\right)$ with $q=1$, and the sequence $u_{k}$ is known to be equiabsolutely integrable.

$\left(\mathrm{GL}_{\infty \infty \infty}\right)$ The same as $\left(\mathrm{GL}_{p q}\right)$ where now $x, x_{k} \in\left(L_{\infty}(G)\right)^{n}, u_{k} \in$ $\left(L_{\infty}(G)\right)^{m}, x_{k}(t) \rightarrow x(t)$ in measure, $\left\|u_{k}\right\|_{\infty} \leqslant L,\|x\|_{\infty},\left\|x_{k}\right\|_{\infty} \leqslant L_{0}, \xi_{k} \rightarrow \xi$ weakly in $\left(L_{1}(G)\right)^{r}$.

$\left(\mathrm{HL}_{q}\right)$ (i) For $1 \leqslant q<\infty, x, x_{k}$ measurable, $x_{k}(t) \rightarrow x(t)$ in measure in $G, u_{k} \in\left(L_{q}(G)\right)^{m},\left\|u_{k}\right\|_{q} \leqslant L$, a constant, and (ii) there are constants $c$ and a function $\psi(t), t \in G, \psi \in L_{1}(G)$, such that for all $(t, x)$, $(t, y) \in A_{G}$ we have $\left|b_{i j}(t, x)\right| \leqslant c,\left|c_{i}(t, x)-c_{i}(t, y)\right| \leqslant \psi(t)$. For $q=1$, we must also require explicitly that the functions $u_{t}(t), t \in G, k=1,2, \ldots$, are equiabsolutely integrable in $G$.

$\left(\mathrm{HL}_{\infty}\right)$ The same as $\left(\mathrm{HL}_{q}\right)$ where now $u_{k} \in\left(L_{\infty}(G)\right)^{m},\left\|u_{k}\right\|_{\infty} \leqslant L$, and still $x, x_{k}$ are measurable and $x_{k}(t) \rightarrow x(t)$ in measure in $G$ as $k \rightarrow \infty$.

Remark 4.3. Lipschitz-type conditions and G-type conditions have been proposed by Browder (Ref. 8) and by Rothe (Ref. 9) in connection with lower semicontinuity theorems for free problems. Analogous conditions have been proposed by Serrin in connection with different modes of convergence. (See Ref. 10 for more bibliographical information.)

\section{Some Preliminary Statements}

We shall follow the notations of Section 2. If $f_{0}(t, x, u)$ and $f(t, x, u)=\left(f_{1}, \ldots, f_{r}\right)$ are given functions on $M$, then, for every $(t, x) \in A$, we shall define sets $Q(t, x) \subset E_{r}$ and $\widetilde{Q}(t, x) \subset E_{r+1}$ as follows:

$$
\begin{aligned}
& Q(t, x)=f(t, x, U(t))=[z \mid z=f(t, x, u), u \in U(t)] \\
& \widetilde{Q}(t, x)=\left[\left(z^{0}, z\right) \mid z^{0} \geqslant f_{0}(t, x, u), z=f(t, x, u), u \in U(t)\right] .
\end{aligned}
$$

Note that here, as well as throughout this paper, $U(t)$ depends only on $t$ 
and not on $x$. Thus, for $x(t), x_{k}(t) \in A(t), u_{k}(t) \in U(t), k=1,2, \ldots$, the differences

$$
\begin{aligned}
& \delta_{k}(t)=f\left(t, x_{k}(t), u_{k}(t)\right)-f\left(t, x(t), u_{k}(t)\right), \quad t \in G, k=1,2, \ldots, \\
& \delta_{k}^{0}(t)=f_{0}\left(t, x_{k}(t), u_{k}(t)\right)-f_{0}\left(t, x(t), u_{k}(t)\right)
\end{aligned}
$$

exist. We have also seen in Sections 3, 4 that, under a variety of Lipschitz-type and growth-type conditions, we have $\delta_{k} \rightarrow 0$ weakly in $\left(L_{1}(G)\right)^{r}$ and $\delta_{k}{ }^{0} \rightarrow 0$ weakly in $L_{1}(G)$ as $k \rightarrow \infty$. These facts are relevant in view of the following statements proved in Ref. 1.

Statement 5.1. A Preliminary Closure Theorem for Mayer Problems. If $G$ is a measurable set of finite measure, and $T_{0}$ has measure zero, if the sets $U(t)$ depend on $t$ only, if $f(t, x, u)$ satisfies continuity property (C), if the corresponding sets $Q(t, x)=f(t, x, U(t))$ defined above are closed and convex for every $(t, x) \in A_{G}-H_{0}$, if $\xi(t), \xi_{k}(t)$, $x(t), x_{k}(t), u_{k}(t), \quad t \in G, \quad k=1,2, \ldots$, are measurable functions, $\xi$, $\xi_{k} \in\left(L_{1}(G)\right)^{r}$, if

$$
\begin{aligned}
& x_{k}(t) \in A(t), \quad u_{k}(t) \in U(t), \quad \xi_{k}(t)=f\left(t, x_{k}(t), u_{k}(t)\right), \quad t \in G, \text { a.e. }, \\
& k=1,2, \ldots \text {, }
\end{aligned}
$$

and $\xi_{k} \rightarrow \xi, \delta_{k} \rightarrow 0$ weakly in $\left(L_{1}(G)\right)^{r}$, and $x_{k}(t) \rightarrow x(t)$ in measure as $k \rightarrow \infty$, then there is a measurable function $u(t), t \in G$, such that

$$
x(t) \in A(t), \quad u(t) \in U(t), \quad \xi(t)=f(t, x(t), u(t)), \quad t \in G \text {, a.e. }
$$

Statement 5.2. A Preliminary Lower Closure Theorem for Lagrange Problems. If $G$ is a measurable set of finite measure, and $T_{0}$ has measure zero, if $f_{0}(t, x, u), f(t, x, u)$ satisfy continuity property (C), if the corresponding subsets $\widetilde{Q}(t, x)$ of $E_{r+1}$ defined above are closed and convex for every $(t, x) \in A_{G}-H_{0}$, if $\xi(t), \xi_{k}(t), \eta_{k}(t), x(t), x_{k}(t), u_{l_{k}}(t)$, $\lambda(t), \lambda_{k}(t), t \in G, k=1,2, \ldots$, are measurable functions, $\xi, \xi_{k} \in\left(L_{1}(G)\right)^{r}$, $\eta_{k}, \lambda, \lambda_{k} \in L_{1}(G)$

$$
\begin{gathered}
x_{k}(t) \in A(t), \quad u_{k_{k}}(t) \in U(t), \quad \xi_{k}(t)=f\left(t, x_{k}(t), u_{k}(t)\right), \\
\eta_{k}(t)=f_{0}\left(t, x_{k}(t), u_{k}(t)\right), \quad t \in G, k=1,2, \ldots,
\end{gathered}
$$

and $\xi_{k} \rightarrow \xi, \delta_{k} \rightarrow 0$ weakly in $\left(L_{1}(G)\right)^{r}, \delta_{k}{ }^{0} \rightarrow 0$ weakly in $L_{1}(G)$, [or $\delta_{k}{ }^{0} \rightarrow 0$ in measure in $\left.G\right], x_{k}(t) \rightarrow x(t)$ in measure as $k \rightarrow \infty,-\infty<i=$ 
$\liminf \int_{G} \eta_{k}(t) d t<+\infty$, and $\eta_{k}(t) \geqslant \lambda_{k}(t), \lambda_{k} \rightarrow \lambda$ weakly in $L_{1}(G)$, then there is a measurable function $U(t), t \in G$, such that

$$
x(t) \in A(t), \quad u(t) \in U(t), \quad \xi(t)=f(t, x(t), u(t)), \quad t \in G, \text { a.e., }
$$

and, if $\eta(t)=f_{0}(t, x(t), u(t)), t \in G$, then $\eta$ is measurable and

$$
\int_{G} \eta(t) d t \leqslant i
$$

Under the sole hypotheses of Statement 5.2, then, $\int_{G} \eta(t) d t$, as a Lebesgue integral, exists (finite, or $-\infty$ ), and (7) holds as stated. Examples show that $\int_{G} \eta(t) d t$ may well be $-\infty$ (see Ref. 1).

In Statement 5.2, the inequality $i>-\infty$ is a consequence of the requirements on the functions $\lambda, \lambda_{k}$. Functions $\lambda, \lambda_{k}$ satisfying these requirements are easily found under mild hypotheses, usually satisfied in applications, under which, furthermore, $\eta$ is certainly of class $L_{1}(G)$, $\int_{G} \eta(t) d t$ is finite, and of course (7) holds as stated. We list here a number of simple hypotheses under which this occurs.

$\left(\alpha_{0}\right)$ There is a real-valued function $\psi(t) \geqslant 0, t \in G, \psi \in L_{1}(G)$, such that $f_{0}(t, x, u) \geqslant-\psi(t)$ for all $(t, x, u) \in M$.

$\left(\beta_{0}\right)$ There is a real-valued function $\psi(t), t \in G, \psi \in L_{1}(G)$, and a constant $\gamma \geqslant 0$ such that $f_{0}(t, x, u) \geqslant-\psi(t)-\gamma|f(t, x, u)|$ for all $(t, x, u) \in M$.

$\left(\gamma_{0}\right) x, x_{k} \in\left(L_{p}(G)\right)^{n},\left\|x_{k}-x\right\|_{p} \rightarrow 0$, for some $p, 1 \leqslant p<\infty$, and there is a real-valued function $\psi(t) \geqslant 0, t \in G, \psi \in L_{1}(G)$, and constants $\gamma$, $\gamma^{\prime} \geqslant 0$ such that $f_{0}(t, x, u) \geqslant-\psi(t)-\gamma|x|^{p}-\gamma^{\prime}|f(t, x, u)|$ for all $(t, x, u) \in M$.

$\left(\delta_{0}\right) x_{k} \in\left(L_{\infty}(G)\right)^{n},\left\|x_{k}\right\|_{\infty} \leqslant L_{1}, \xi_{k} \in\left(L_{\infty}(G)\right)^{r},\left\|\xi_{k}\right\|_{\infty} \leqslant L_{2}$ for given constants $L_{1}, L_{2}$ and there are a real-valued function $\psi(t) \geqslant 0$, $t \in G, \psi \in L_{1}(G)$, and a real-valued monotone nondecreasing function $\sigma(\xi), 0 \leqslant \xi<+\infty$, such that $f_{0}(t, x, u) \geqslant-\psi(t) \sigma(|x|+|f(t, x, u)|)$ for all $(t, x, u) \in M$.

The proofs of Statements 5.1 and 5.2 (given in Ref. 1) are very simple, and depend on the fact that, if $\delta_{k} \rightarrow 0$ weakly, and $\xi_{k}(t)=$ $f\left(t, x_{k}(t), u_{k}(t)\right)$ converges weakly to $\hat{\xi}(t)$, then for the auxiliary functions

$$
\xi_{k t}(t)=f\left(t, x(t), u_{k}(t)\right)=\xi_{k}(t)-\delta_{k}(t), \quad t \in G, \quad k=1,2, \ldots,
$$

we have $\xi_{k} \rightarrow \xi$ weakly. Analogously, if $\delta_{k}{ }^{0} \rightarrow 0$ weakly, and $\eta_{k}(t)=$ $f_{0}\left(t, x_{k}(t), u_{k}(t)\right)$, then

$$
\begin{gathered}
\bar{\eta}_{k}(t)=f_{0}\left(t, x(t), u_{k}(t)\right)=\eta_{k}(t)-\delta_{k}^{0}(t), \quad t \in G, k=1,2, \ldots, \\
\liminf _{k \rightarrow \infty} \int_{G} \bar{\eta}_{k}(t) d t=\liminf _{k \rightarrow \infty} \int_{G} \eta_{k}(t) d t .
\end{gathered}
$$


Details are given in Ref. 1. Alternatively, if $\delta_{k}{ }^{0} \rightarrow 0$ in measure in $G$ as $k \rightarrow \infty$, then there is a subsequence, say still [k], such that $\delta_{k}{ }^{0}(t) \rightarrow 0$ a.e. in $G$. We shall now consider the functions $\eta_{N}^{(s)}(t), \eta^{(s)}(t), \eta(t), t \in G$, defined in Ref. 1 , Section 5 , in terms of the given elements $\eta_{k}$ and the operation of lim inf, and the analogous functions $\bar{\eta}_{N}^{(s)}(t), \bar{\eta}^{(s)}(t), \bar{\eta}(t)$, $t \in G$, defined in terms of the elements $\bar{\eta}_{k}$. Since $\delta_{k}{ }^{0}(t)=\eta_{k}(t)-\bar{\eta}_{k}(t)$, $t \in G$, and $\delta_{k}{ }^{0}(t) \rightarrow 0$ as $k \rightarrow \infty$ pointwise a.e. in $G$, we derive that $\bar{\eta}^{(s)}(t)=\eta^{(s)}(t), \vec{\eta}(t)=\eta(t)$ a.e. in $G$.

\section{Closure Theorems for Mayer Problems}

For the sake of brevity, when referring below to conditions $\left(\mathrm{F}_{p}\right)$, $1 \leqslant p \leqslant \infty$, of Section 4 , we shall mean $\left(F_{p}\right)$ if $1 \leqslant p<\infty$, and $\left(F_{\infty}\right)$ if $p=\infty$. Analogous convention holds for $\left(\mathrm{G}_{p q}\right)$ and $\left(\mathrm{H}_{q}\right)$.

Theorem 6.1. Let $G$ be a measurable set of finite measure, $T_{0} \subset G$ of measure zero, and let $f(t, x, u)$ satisfy the Carathéodory continuity condition (C) on $M=\{(t, x, a)\}$. Let the sets $Q(t, x)=$ $f(t, x, U(t))$ be convex and closed for $(t, x) \in A_{G}-H_{0}$. Let $\xi(t), x(t)$, $\xi_{l}(t), x_{k}(t), u_{k}(t), t \in G, k=1,2, \ldots$, be measurable functions, satisfying

$$
\begin{array}{r}
x_{k}(t) \in A(t), \quad u_{k}(t) \in U(t), \quad \xi_{k}(t)=f\left(t, x_{k}(t), u_{k}(t)\right), \quad t \in G, \text { a.e. }, \\
k=1,2, \ldots,
\end{array}
$$

$\xi, \xi_{k} \in\left(L_{1}(G)\right)^{r}, \xi_{k} \rightarrow \xi$ weakly, and let one of the following conditions (a), (b), (c) hold:

(a) For some $p, 1 \leqslant p \leqslant \infty, x, x_{k} \in\left(L_{p}(G)\right)^{n}$ and condition $\left(\mathrm{F}_{p}\right)$ holds; $\left(\phi\right.$ as in $\left.\mathrm{G}_{p q}\right)$.

(b) For some $p, q, 1 \leqslant p, q \leqslant \infty, x, x_{k} \in\left(L_{p}(G)\right)^{n}$ and $\phi\left(u_{k}\right) \in$ $\left(L_{q}(G)\right)^{m}$ and condition $\left(\mathrm{G}_{p q}\right)$ holds.

(c) For some $q, 1 \leqslant q \leqslant \infty, u_{k} \in\left(L_{q}(G)\right)^{m}$ and condition $\left(\mathrm{H}_{q}\right)$ holds. Then, there is a measurable function $u(t), t \in G$ such that

$$
x(t) \in A(t), \quad u(t) \in U(t), \quad \xi(t)=f(t, x(t), u(t)), \quad t \in G, \text { a.e. }
$$

Proof. Under any of the conditions (a), (b), (c), we have seen that $\left\|\delta_{k}\right\|_{1} \rightarrow 0$, and the theorem now follows from Statement 5.1 and the remarks at the end of that section.

Remark 6.1. Under the sole hypotheses of Theorem 6.1, parts 
(b) and (c), it may well occur that the measurable control $u(t), t \in G$, whose existence is proved in parts (b) and (c), is not of class $\left(L_{q}(G)\right)^{m}$, or $\|u\|_{q}$ is not $\leqslant L$. The first one of these occurrences is shown by the following example. Take $v=1, n=2, p=\infty, 1 \leqslant q<2, A=$ $[0,1] \times E_{2}, U=[(u, v) \mid 0 \leqslant u \leqslant 1,0 \leqslant v<+\infty]$, a fixed closed convex set, $M=A \times U$, and $f(t, u, v)=\left(f_{1}, f_{2}\right), f_{1}(t, u, v)=u$, $f_{2}(t, u, v)=[1-4 u(1-u)] v+4 u(1-u) v^{1 / 2}$, so that $f$ is continuous on $M$. Here we have $f(t, 0, v)=(0, v), f(t, 1, v)=(1, v), f(t, 1 / 2, v)=$ $\left(1 / 2, v^{1 / 2}\right)$. On the other hand, $Q=f(t, U)$ is the fixed closed convex set $Q=\left[\left(z^{1}, z^{2}\right) \mid 0 \leqslant z^{1} \leqslant 1,0 \leqslant z^{2}<+\infty\right]$. We take $x_{k}(t)=t-i / 2 k$, $u_{k}(t)=1$ for $i / k \leqslant t<i / k+1 / 2 k, x_{k}(t)=(i+1) / 2 k, u_{k}(t)=0$ for $i / k+1 / 2 k \leqslant i<(i+1) \mid k, i=0,1, \ldots, k-1$, and we take $y_{k}(t)=2 t^{1 / 2}$, $\xi_{k}{ }^{1}(t)=x_{k}{ }^{\prime}(t), \xi_{k}{ }^{2}(t)=y_{k}{ }^{\prime}(t)=v_{k}(t)=t^{-1 / 2}, 0 \leqslant t \leqslant 1, k=1,2, \ldots$ Also, we take $x(t)=t / 2, \quad y(t)=2 t^{1 / 2}, \quad \xi^{1}(t)=x^{\prime}(t)=1 / 2, \quad \xi^{2}(t)=$ $y^{\prime}(t)=t^{-1 / 2}, 0 \leqslant t \leqslant 1$, so that $x_{k} \rightarrow x$ uniformly, $y_{k}=y, \xi_{k}{ }^{2}=\xi^{2}$, $\xi_{k}{ }^{1} \rightarrow \xi^{1}$ weakly in $L_{1},\left(u_{k}, v_{k}\right) \in L_{q}[0,1],\left\|u_{k}\right\|_{q}=2^{-1 / q},\left\|v_{k}\right\|_{q}=$ $2(2-q)^{-1}$. Now the equations $1 / 2=f_{1}(t, u, v), t^{-1 / 2}=f_{2}(t, u, v)$ yield $u(t)=1 / 2, v(t)=t^{-1}, 0<t \leqslant 1$, and $v$ is not even $L$-integrable in [0, 1$]$.

Remark 6.2. In certain situations of actual interest, it is possible to guarantee that the control $u$, whose existence is proved in Theorem 6.1, parts (b) and (c), belongs to $\left(L_{q}(G)\right)^{m}$ and $\|u\|_{q} \leqslant L$. Here are some statements in this direction. In Section 7 below, we shall consider the case with $f$ linear in $u$.

Theorem 6.2. The same as Theorem 6.1 with conditions (b) or (c), with $\phi(u)=u, 1 \leqslant p, q \leqslant \infty$, and in addition we assume that there is a function $\Phi(t) \geqslant 0, t \in G, \Phi \in L_{q}(G)$, such that for every $t \in G$ and $u \in U(t)$ we have $|u| \leqslant \Phi(t)$, and we take $L=\|\Phi\|_{q}$. Then there exists a function $u \in\left(L_{q}(G)\right)^{m},\|u\|_{q} \leqslant L$, such that (9) holds.

Proof. Indeed, here all measurable functions $u(t), t \in G$, with $u(t) \in U(t)$, satisfy the relation $|u(t)| \leqslant \Phi(t), t \in G$ (a.e.), hence $u \in\left(L_{q}(G)\right)^{m},\|u\|_{q} \leqslant L_{1}$. Theorem 6.2 is now a simple corollary of Theorem 6.1.

Theorem 6.3. The same as Theorem 6.1, with condition (b), with $\phi(u)=u, 1 \leqslant p, q \leqslant \infty$, where in addition we know that condition $\left(\mathrm{G}_{p q}^{*}\right)$ holds and there is a function $\Phi_{0}(t) \geqslant 0, t \in G, \Phi_{0} \in L_{q}(G)$, and constants $\gamma, \gamma^{\prime} \geqslant 0$ such that for every $(t, x) \in A_{G}-H_{0}, z \in Q(t, x)$, 
there is some $u \in U(t)$ such that $|u|^{q} \leqslant \Phi_{0}(t)+\gamma|x|^{p}+\gamma^{\prime}|z|$, and also we know that $c^{\prime} y^{\prime}<1$ and

$$
L^{a}=\left(1-c^{\prime} \gamma^{\prime}\right)^{-1}\left[\left(C_{0}+\gamma^{\prime} C\right)+\left(\gamma+c \gamma^{\prime}\right) L_{0}^{p}\right],
$$

where $C=\int_{G} \psi(t) d t, C_{0}=\int_{G} \Phi_{0}(t) d t$.

Theorem 6.4. The same as Theorem 6.1, with condition (c), with $1 \leqslant q \leqslant \infty$, and the same further requirement as above with $\gamma=0$.

In either case, there is a function $u \in\left(L_{q}(G)\right)^{m},\|u\|_{q} \leqslant L$, such that (9) holds.

Proof. Let us prove first that for every measurable function $\xi(t)=\left(\xi^{1}, \ldots, \xi^{r}\right), t \in G, \xi(t) \in Q(t, x(t))$ a.e. in $G$, there is also a measurable function $u(t)=\left(u^{1}, \ldots, u^{m}\right), t \in G, u(t) \in U(t)$ a.e. in $G$, such that $\xi(t)=f(t, x(t), u(t))$, and $|u(t)|^{q} \leqslant \Phi_{0}(t)+\gamma|x(t)|^{p}+\gamma^{\prime}|\xi(t)|, t \in G$ a.e.). For every $\epsilon>0$, there is a compact subset $K$ of $G$ on which $\xi(t), x(t), \Phi_{0}(t)$ are continuous, and $|G-K|<\epsilon$. For every $t \in K$, let $J(t)$ denote the nonempty set of all $u \in U(t)$ such that $f(t, x(t), u)=$ $\xi(t),|u|^{\alpha} \leqslant \Phi_{0}(t)+\gamma|x(t)|^{p}+\gamma^{\prime}|\xi(t)|$. Since $\xi, x, \Phi_{0}$ are continuous on $K$, and $f(t, x, u)$ is continuous on the closed set $M_{K}$, and hence $f(t, x(t), u)$ is continuous on the closed set $[(t, x, u) \mid t \in K, x=x(t)$, $u \in U(t)]$, we conclude that, for every $t \in K$, the set $J(t)$ is closed and compact, and that the set $J_{K}=\left[\left.(t, x, u)|t \in K, x=x(t)| u\right|^{q,} \leqslant \Phi_{0}(t)+\right.$ $\left.\gamma|x(t)|^{p}+\gamma^{\prime}|\xi(t)|, f(t, x(t), u)=\xi(t)\right]$ is compact. By implicit function theorem (Ref. 11), there is a measurable function $u(t), t \in K, u(t) \in J(t)$, such that $f(t, x(t), u(t))=\xi(t), t \in K$, and $|u(t)|^{\alpha} \leqslant \Phi_{0}(t)+\gamma|x(t)|^{p}+$ $\gamma^{\prime}|\xi(t)|, t \in K$. Since $|G-K|<\epsilon$, we conclude that such a measurable function $u(t)$ can be defined a.e. in $G$. Now we have, with the usual notations,

$$
\left|\xi_{k}(t)\right|=\left|f\left(t, x(t), u_{l k}(t)\right)\right| \leqslant \psi(t)+c|x(t)|^{p}+c^{t}\left|u_{k}(t)\right|^{q},
$$

hence $\left\|\xi_{k}\right\|_{1} \leqslant C+c L_{0}{ }^{p}+c^{\prime} L^{q}$, where $\xi_{k} \rightarrow \xi$ weakly in $\left(L_{1}(G)\right)^{r}$. Hence,

$$
\|\xi\|_{1} \leqslant C+c L_{0}^{p}+c^{\prime} L^{q} \text {. }
$$

On the other hand, we have

and finally

$$
\|u\|_{u}^{\alpha} \leqslant C_{0}+\gamma L_{0}^{p}+\gamma^{\prime}\|\xi\|_{1}
$$

$$
\begin{aligned}
\|u\|_{a}^{q} & \leqslant C_{0}+\gamma L_{0}^{p}+\gamma^{\prime}\left(C+c L_{0}^{p}+c^{\prime} L^{q}\right) \\
& =\left(C_{0}+\gamma^{\prime} C\right)+\left(\gamma+c \gamma^{\prime}\right) L_{0}^{p}+c^{\prime} \gamma^{\prime} L^{q}=L^{q}
\end{aligned}
$$


We have proved that there is a function $u$ as in Theorem 6.1 with $u \in\left(L_{q}(G)\right)^{m},\|u\|_{q} \leqslant L_{1}$.

It is easy to see that in the present situation we have

$$
\begin{gathered}
|\xi(t)| \leqslant \psi(t)+c|x(t)|^{p}+c^{\prime}|u(t)|^{q}, \\
|u(t)|^{q} \leqslant \Phi_{0}(t)+\gamma|x(t)|^{p}+\gamma^{\prime}|\xi(t)|,
\end{gathered}
$$

and hence,

$$
\begin{aligned}
|\xi(t)| & \leqslant\left(1-c^{\prime} \gamma^{\prime}\right)^{-1}\left(\psi(t)+c^{\prime} \Phi_{0}(t)+\left(c+c^{\prime} \gamma\right)|x(t)|^{p}\right), \\
|u(t)|^{q} & \leqslant\left(1-c^{\prime} \gamma^{\prime}\right)^{-1}\left(\Phi_{0}(t)+\gamma^{\prime} \psi(t)+\left(\gamma+c \gamma^{\prime}\right)|x(t)|^{p}\right) .
\end{aligned}
$$

\section{Two Closure Theorems for Mayer Problems with $f$ Linear in $u$}

Theorem 7.1. (a) If $G$ is a measurable set of finite measure, $T_{0}$ has measure zero, if $f(t, x, u)=\left(f_{1}, \ldots, f_{r}\right)$ satisfy continuity condition (C) on the set $M$, if the sets $U(t)$ are convex for all $t \in G-T_{0}$, if $f(t, x, u)$ is linear in $u$, that is, of the form $f(t, x, u)=B(t, x) u+C(t, x)$, $B=\left[b_{i j}(t, x)\right], C=\left[c_{i}(t, x)\right]$, matrices of the types $r \times m, r \times 1$, if for some $p, q, 1 \leqslant p, q \leqslant \infty$, condition ( $\mathrm{GL}_{p q}$ ) holds, if $\xi_{k}(t), x(t), x_{k}(t)$, $u(t), u_{k}(t), t \in G, k=1,2, \ldots$, are measurable functions $\xi_{k} \in\left(L_{1}(G)\right)^{r}$, $x, \quad x_{k} \in\left(L_{p}(G)\right)^{n}, \quad u, \quad u_{k} \in\left(L_{q}(G)\right)^{m}, \quad x_{k}(t) \in A(t), \quad u_{k}(t) \in U(t), \quad \xi_{k}(t)=$ $f\left(t, x_{k}(t), u_{k}(t)\right), t \in G$, (a.e.), $k=1,2, \ldots$, and $x_{k} \rightarrow x$ strongly in $\left(L_{p}(G)\right)^{n}$, $u_{k} \rightarrow u$ weakly in $\left(L_{q}(G)\right)^{m}$, then $x(t) \in A(t), u(t) \in U(t)$, $t \in G$, (a.e.), and if $\xi(t)=f(t, x(t), u(t)), t \in G$, then $\xi \in\left(L_{1}(G)\right)^{r}$, and $\xi_{k} \rightarrow \xi$ weakly in $\left(L_{1}(G)\right)^{r}$.

(b) If for some $q, 1 \leqslant q \leqslant \infty$, condition $\left(\mathrm{HL}_{q}\right)$ holds, $x, x_{k}$ are only measurable, $x_{k}(t) \rightarrow x(t)$ in measure in $G$ as $k \rightarrow \infty, u_{k} \rightarrow u$ weakly in $\left(L_{q}(G)\right)^{m}$, then as before $\xi \in\left(L_{1}(G)\right)^{r}, \xi_{k} \rightarrow \xi$ weakly in $\left(L_{1}(G)\right)^{r}$ as $k \rightarrow \infty$.

Note that, in Theorem 7.1, the requirement $\xi_{k} \rightarrow \xi$ weakly in $\left(L_{1}(G)\right)^{r}$ is deleted from the hypotheses $\left(\mathrm{GL}_{p q}\right)$ or $\left(\mathrm{H}_{q}\right)$, and is proved instead as a consequence of the hypothesis $u_{k} \rightarrow u$ weakly in $\left(L_{1}(G)\right)^{m}$.

Proof. Note that $u_{k}(t) \in U(t), t \in G$, (a.e.), $k=1,2, \ldots$, where, for almost all $t \in G$, the sets $U(t)$ are convex by hypothesis, and closed as a consequence of property (C). Since $u_{k} \rightarrow u$ weakly in $L_{q}(G), 1 \leqslant q \leqslant \infty$, we conclude that $u(t) \in U(t), t \in G$, (a.e.) by force of Statement 5.1 applied to the auxiliary function $g=u$, or $g_{i}=u^{i}, i=1, \ldots, m$. Since the sets $A(t)$ are closed for almost all $t \in G$, again as a consequence of property (C), we derive as usual that $x(t) \in A(t), t \in G$, (a.e.). Thus, 
$\xi(t)=f(t, x(t), u(t))$ exists and is certainly measurable in $G$. On the other hand, by property $\left(\mathrm{GL}_{p q}\right)$, the differences

$$
\begin{aligned}
\delta_{k}(t) & =\xi_{k}(t)-\xi_{k}(t)=f\left(t, x_{k}(t), u_{k}(t)\right)-f\left(t, x(t), u_{k}(t)\right) \\
& =\left[B\left(t, x_{k i}(t)\right)-B(t, x(t))\right] u_{k}(t)+\left[C\left(t, x_{k}(t)\right)-C(t, x(t))\right]
\end{aligned}
$$

are of class $\left(L_{1}(G)\right)^{r}$, and $\left\|\delta_{k}\right\|_{1} \rightarrow 0$ as $k \rightarrow \infty$. [Note that the functions $u_{k_{k}}$ are certainly equiabsolutely integrable in $G$ since $u_{k} \rightarrow u$ weakly in $\left(L_{q}(G)\right)^{m}, 1 \leqslant q \leqslant \infty$, hence equibounded in the $L_{q}$ norms, and then by the use of Hölder inequality for $1<q<\infty$, Dunford-Pettis theorem for $q=1$, and trivial estimates for $q=\infty$.] Finally,

$$
\delta_{k}{ }^{\prime}(t)=\bar{\xi}_{k}(t)-\xi(t)=B(t, x(t))\left[u_{k}(t)-u(t)\right], \quad t \in G, k=1,2, \ldots,
$$

where now the entries of the matrix $B$ are fixed bounded measurable functions, and $\bar{\xi}_{k} \in\left(L_{1}(G)\right)^{r}, u, u_{k} \in\left(L_{q}(G)\right)^{m}, u_{k} \rightarrow u$ weakly in $\left(L_{q}(G)\right)^{m}$. Then, $\xi \in\left(L_{1}(G)\right)^{r}$, and $\delta_{k}{ }^{\prime} \rightarrow 0$ weakly in $\left(L_{q}(G)\right)^{r}$, and hence also weakly in $\left(L_{1}(G)\right)^{r}$. From $\xi_{k}-\xi=\delta_{k}+\delta_{k}{ }^{\prime}$ we conclude that $\xi_{k} \rightarrow \xi$ weakly in $\left(L_{1}(G)\right)^{r}$.

Thus, $u$ is measurable, $\xi \in\left(L_{1}(G)\right)^{r}$, and $x(t) \in A(t), u(t) \in U(t)$, $\xi(t)=f(t, x(t), u(t)), t \in G$ (a.e.). We have proved (a) of Theorem 7.1. Note that we have just proved here (9) directly, and thus we did not need to use Statement 5.1 for $\xi(t)$.

The proof of part (b) is analogous.

Theorem 7.2. (a) The same as Theorem 7.1 with $1 \leqslant p \leqslant \infty$, $1<q \leqslant \infty$, the sets $U(t)$ convex for almost all $t \in G, f(t, x, u)$ linear in $u$, that is, of the form $f(t, x, u)=B(t, x) u+C(t, x)$, satisfying conditions $(C)$ and $\left(\mathrm{G}_{p q}\right)$, and where $\xi, \xi_{k} \in\left(L_{1}(G)\right)^{r}, x, x_{k} \in\left(L_{p}(G)\right)^{n}, u_{k} \in$ $\left(L_{q}(G)\right)^{m},\|x\|_{p},\left\|x_{k}\right\|_{p} \leqslant L_{0},\left\|u_{k}\right\|_{p} \leqslant L, \xi_{k} \rightarrow \xi$ weakly in $\left(L_{1}(G)\right)^{r}$, $x_{k}(t) \rightarrow x(t)$ in measure in $G$ as $k \rightarrow \infty$. Then, there is a function $u \in\left(L_{q}(G)\right)^{m}$ with $\|u\|_{q} \leqslant L$ such that (9) holds.

(b) The same as above, where conditions (C) and $\left(\mathrm{HL}_{q}\right)$ hold, $\xi, \xi_{k} \in\left(L_{1}(G)\right)^{r}, x, x_{k}$ are only measurable, $u_{k} \in\left(L_{q}(G)\right)^{m},\left\|u_{k}\right\|_{q} \leqslant L$, $\xi_{k} \rightarrow \xi$ weakly in $\left(L_{1}(G)\right)^{r}$, and $x_{k}(t) \rightarrow x(t)$ in measure in $G$ as $k \rightarrow \infty$. Then, there is a function $u \in\left(L_{q}(G)\right)^{m}$, with $\|u\|_{q} \leqslant L$ such that (9) holds.

Proof. Since $\left\|u_{k}\right\|_{q} \leqslant L, k=1,2, \ldots$, where now $1<q \leqslant \infty$, there are a subsequence, say still $u_{k}$ for the sake of simplicity, and a function $u \in\left(L_{q}(G)\right)^{m}$ such that $u_{k} \rightarrow u$ weakly in $\left(L_{q}(G)\right)^{m}$. The proof is now similar to the one for Theorem 7.1. 


\section{Lower Closure Theorems for Lagrange Problems}

Theorem 8.1. Let $G$ be a measurable set of finite measure, let $T_{0} \subset G$ of measure zero, and let $f_{0}(t, x, u), f(t, x, u)=\left(f_{1}, \ldots, f_{r}\right)$ satisfy condition (C). Let the corresponding sets $\bar{Q}(t, x)$ defined in Section 5 be convex and closed for every $(t, x) \in A_{G}-H_{0}$. Let $\lambda(t)$, $\lambda_{k}(t), \xi(t), x(t), \xi_{k}(t), \eta_{k}(t), x_{k}(t), u_{k}(t), t \in G, k=1,2, \ldots$, be measurable functions, with

$$
\begin{gathered}
x_{k}(t) \in A(t), \quad u_{k}(t) \in U(t), \quad \xi_{k}(t)=f\left(t, x_{k}(t), u_{k}(t)\right), \\
\eta_{k}(t)=f_{0}\left(t, x_{k}(t), u_{k_{k}}(t)\right), \quad t \in G, \text { a.e., } \quad k=1,2, \ldots, \\
\xi_{k}, \xi \in\left(L_{1}(G)\right)^{r}, \quad \eta_{k}, \lambda, \lambda_{k} \in L_{1}(G), \quad \eta_{k}(t) \geqslant \lambda_{k}(t), \\
\xi_{k} \rightarrow \xi \text { weakly in }\left(L_{1}(G)\right)^{r}, \quad \lambda_{k} \rightarrow \lambda \text { weakly in } L_{1}(G), \\
-\infty<i=\lim \inf \int_{G} \eta_{k}(t) d t<+\infty .
\end{gathered}
$$

Let one of the following conditions (a), (b), or (c) hold:

(a) for some $p, 1 \leqslant p \leqslant \infty, x, x_{k} \in\left(L_{p}(G)\right)^{n}$ and condition $\left(\mathrm{F}_{p}\right)$ holds (for both $f_{0}$ and $f$ ),

(b) for some $p, q, 1 \leqslant p, q \leqslant \infty, x, x_{k} \in\left(L_{p}(G)\right)^{n}, \phi\left(u_{l_{2}}\right) \in\left(L_{q}(G)\right)^{m}$ and condition $\left(\mathrm{G}_{p q}\right)$ holds (for both $f_{0}$ and $f$ ), ( $\phi$ as in $\mathrm{G}_{p q}$ ),

(c) for some $q, 1 \leqslant q \leqslant \infty, u_{k} \in\left(L_{q}(G)\right)^{m}$ and condition $\left(\mathrm{H}_{q}\right)$ holds (for both $f_{0}$ and $f$ ).

Then, there is a measurable function $u(t), t \in G$, such that

$$
\begin{gathered}
x(t) \in A(t), \quad u(t) \in U(t), \quad \xi(t)=f(t, x(t), u(t)), \quad t \in G, \text { a.e., } \\
\int_{G} \eta(t) d t \leqslant \liminf _{k \rightarrow \infty} \int_{G} \eta_{k}(t) d t
\end{gathered}
$$

where $\eta(t)=f_{0}(t, x(t), u(t)), t \in G$.

Here $\int_{G} \eta(t) d t$, as a Lebesgue integral, exists (finite, or $-\infty$ ). Under any of the conditions $\left(\alpha_{0}\right),\left(\beta_{0}\right),\left(\gamma_{0}\right),\left(\delta_{0}\right)$ of Section 5 , certainly functions $\lambda, \lambda_{k}$ satisfying the above requirements are easily found, $\eta \in L_{1}(G)$, and $\int_{G} \eta(t) d t$ is finite and $\leqslant i$.

Proof. Under any of the conditions (a), (b), (c), we have seen in Section 4 that $\left\|\delta_{k}\right\|_{1} \rightarrow 0,\left\|\delta_{k}^{0}\right\|_{1} \rightarrow 0$, and the theorem follows from Statement 5.2 and the remarks at the end of that section. 
Under (b) and (c), $x_{k}$ and $u_{k}$ are bounded in measure [that is, satisfy (ii) of Remark 3.1] and (by that remark) we know that $\delta_{k}^{0}(t) \rightarrow 0$ in measure in $G$; and because of the alternate hypothesis in Statement 5.2, we need not assume that $f_{0}$ satisfies the remaining requirements in $\left(\mathrm{G}_{p q}\right)$ or $\left(\mathrm{H}_{q}\right)$. This holds also for the remaining statements below.

Under the sole hypotheses of Theorem 8.1, parts (b) and (c), with $\phi(u)=u$, it may well occur that the measurable control $u(t), t \in G$, whose existence is proved in parts (b) and (c), is not of class $\left(L_{q}(G)\right)^{m}$, or $\|u\|_{q}$ is not $\leqslant L$, as the $u_{k}$ are. The example we have already seen in Remark 6.1 suffices. In certain situations of actual interest it is possible to guarantee that the control $u$, in parts (b) and (c), belongs to $\left(L_{q}(G)\right)^{m}$ and $\|u\|_{q} \leqslant L$. Below are some of the cases, similar to those we have considered in Section 6.

Theorem 8.2. The same as Theorem 8.1 with $\phi(u)=u, 1 \leqslant p$, $q \leqslant \infty$, where in addition there is a function $\Phi(t) \geqslant 0, t \in G, \Phi \in L_{q}(G)$, such that for every $t \in G$ and $u \in U(t)$ we have $|u| \leqslant \Phi(t)$ and we take $L=\|\Phi\|_{q}$. Then the control $u$ of Theorem 8.1 is of class $\left(L_{q}(G)\right)^{m}$ and $\|u\|_{q} \leqslant L$.

A corollary of Theorem 8.1 with the remarks we have seen for Theorem 6.2.

Theorem 8.3. The same as Theorem 8.1 with condition (b), $\phi(u)=u$, and the additional requirements stated in Theorem 6.3. Then, the control $u$ of Theorem 8.1 is of class $\left(L_{q}(G)\right)^{m}$ and $\|u\|_{q} \leqslant L$.

A corollary of Theorem 8.1 with the remarks we have seen for Theorem 6.3.

\section{Two Lower Closure Theorems for Lagrange Problems with $f$ Linear in $u$}

Theorem 9.1. (a) If $G$ is a measurable set of finite measure, and $T_{0}$ has measure zero, if $f_{0}(t, x, u), f(t, x, u)=\left(f_{1}, \ldots, f_{r}\right)$ satisfy continuity condition (C) on the set $M$, if the sets $U(t)$ are convex for all $t \in G-T_{0}$, if $f_{0}(t, x, u)$ is convex with respect to $u$ for every $(t, x) \in$ $A_{G}-H_{0}$, and $f(t, x, u)$ is linear in $u$, that is, of the form $f(t, x, u)=$ $B(t, x) u+C(t, x), B=\left[b_{i j}(t, x)\right], C=\left[c_{i}(t, x]\right)$, matrices of the types $r \times m, r \times 1$, if $f_{0}$ satisfies condition $\left(\mathrm{G}_{p q}^{*}\right)$ with $\phi(u)=u$ and $f$ condition $\left(\mathrm{GL}_{p q}\right)$ for some $p, q, 1 \leqslant p, q \leqslant \infty$, if $\xi_{k}(t), \eta_{k}(t), x_{k}(t), u_{k}(t), t \in G$, 
$k=1,2, \ldots$, are measurable functions, $\xi_{k} \in\left(L_{1}(G)\right)^{r}, \quad \eta_{k} \in L_{1}(G), x$, $x_{k} \in\left(L_{p}(G)\right)^{n}, u, u_{l k} \in\left(L_{q}(G)\right)^{m}$,

$$
\begin{aligned}
& x_{k}(t) \in A(t), \quad u_{k}(t) \in U(t), \quad \xi_{k}(t)=f\left(t, x_{k}(t), u_{k}(t)\right), \\
& \eta_{k}(t)=f_{0}\left(t, x_{k}(t), u_{k}(t)\right), \quad t \in G, \text { a.e., } \quad k=1,2, \ldots,
\end{aligned}
$$

and $x_{i}(t) \rightarrow x(t)$ in measure, $u_{k} \rightarrow u$ weakly in $\left(L_{q}(G)\right)^{m}$ as $k \rightarrow \infty$, then $x(t) \in A(t), u(t) \in U(t), t \in G$, a.e., and, if $\xi(t)=f(t, x(t), u(t)), \eta(t)=$ $f_{0}(t, x(t), u(t)), t \in G$, then $\xi \in\left(L_{1}(G)\right)^{r}, \eta \in L_{1}(G), \xi_{k} \rightarrow \xi$ weakly in $\left(L_{1}(G)\right)^{r}$, and

$$
\int_{G} \eta(t) d t \leqslant i=\lim _{k \rightarrow \infty} \int_{G} \eta_{k}(t) d t
$$

(b) The same as (a), where now $f_{0}$ satisfies condition $\left(\mathrm{H}_{q}{ }^{*}\right)$ and $f$ condition $\left(\mathrm{HL}_{q}\right)$ for some $q, 1 \leqslant q \leqslant \infty, \xi_{k} \in\left(L_{1}(G)\right)^{r}, x, x_{k}$ are only measurable, $u, u_{k} \in\left(L_{q}(G)\right)^{m}, x_{k}(t) \rightarrow x(t)$ in measure, $u_{k} \rightarrow u$ weakly in $\left(L_{q}(G)\right)^{m}$ as $k \rightarrow \infty$.

Proof. As in the proof of Theorem 7.1, we know that $f_{0}, f$ satisfy property $(\mathscr{D})$, that $x(t) \in A(t), u(t) \in U(t), t \in G$ (a.e.), and finally, $\xi \in\left(L_{p}(G)\right)^{r}$. If $\bar{\xi}_{k}(t)=f\left(t, x(t), u_{k}(t)\right), \bar{\eta}_{k}(t)=f_{0}\left(t, x(t), u_{k}(t)\right), \quad t \in G$, and $\delta_{k}(t)=\xi_{k}(t)-\bar{\xi}_{k}(t), \delta_{k}{ }^{0}=\eta_{k}(t)-\bar{\eta}_{k}(t), t \in G$, then we know from Section 4, Part B, that $\delta_{k} \rightarrow 0$ strongly in $\left(L_{1}(G)\right)^{r}, \delta_{k}{ }^{0} \rightarrow 0$ strongly in $L_{1}(G)$. As in the proof of Theorem 7.1, we know that $\xi_{k} \rightarrow \xi$ weakly in $\left(L_{1}(G)\right)^{r}$. Finally, (13) follows from the lower semicontinuity Theorem 10.1 below.

Note that we had the measurable function $u$ above and we found $\xi \in\left(L_{1}(G)\right)^{r}, \eta \in L_{1}(G)$, with $x(t) \in A(t), u(t) \in U(t), \xi(t)=f(t, x(t), u(t))$, $\eta(t)=f_{0}(t, x(t), u(t)), t \in G$ (a.e.), satisfying $x(t) \in A(t), u(t) \in U(t)$, $\int_{G} \eta(t) d t \leqslant i$. Actually, we prove the last relation by using Theorem 10.1 . We cannot use Theorem 8.1 here since this statement only proves the existence of some $\bar{u}$ having analogous properties, but we do not know whether $\bar{u}=u$.

Theorem 9.2. The same as Theorem 8.1 with $1 \leqslant p \leqslant \infty$, $1<q \leqslant \infty$, the sets $U(t)$ convex for all $t \in G-T_{0}, f_{0}(t, x, u)$ convex in $u$ for all $(t, x) \in A_{G}-H_{0}, f(t, x, u)$ linear in $u$, that is, of the form $f(t, x, u)=B(t, x) u+C(t, x)$, both $f_{0}$ and $f$ satisfying continuity condition $(\mathrm{C})$, where $f_{0}$ satisfies condition $\left(\mathrm{G}_{p q}\right)$, or $\left(\mathrm{H}_{q}\right)$, and $f$ satisfies condition $\left(\mathrm{GL}_{p q}\right)$ or $\left(\mathrm{HL}_{q}\right)$.

Note that the sets $Q(t, x)$ are necessarily convex and closed for almost all $t \in G$ and all $x \in A(t)$ under the conditions of Theorem 9.2. 
The proof of this statement is the same as for Theorem 8.1 with the remarks made for Theorem 9.1 .

\section{Lower Semicontinuity Theorems}

Theorem 10.1. (a) If $G$ is a measurable set of finite measure, and $T_{0}$ has measure zero, if the sets $U(t)$ are convex for $t \in G-T_{0}$, and $f_{0}(t, x, u)$ satisfies conditions $(C)$ and $\left(F_{p} *\right)$ for some $1 \leqslant p \leqslant \infty$, and is convex with respect to $u$ for every $(t, x) \in A_{G}-H_{0}$, if $x(t), u(t), x_{k}(t)$, $u_{k}(t), \eta_{k}(t), t \in G$, are measurable functions, with $x_{k}(t) \in A(t), u_{k}(t) \in U(t)$, $\eta_{k}(t)=f_{0}\left(t, x_{k}(t), u_{k}(t)\right), t \in G$ (a.e.), $k=1,2, \ldots$, and $x, x_{k} \in\left(L_{p}(G)\right)^{n}$, $u, u_{k} \in\left(L_{1}(G)\right)^{m}, \eta_{k} \in L_{1}(G), x_{k} \rightarrow x$ strongly in $\left(L_{p}(G)\right)^{n}, u_{k} \rightarrow u$ weakly in $\left(L_{1}(G)\right)^{m}$, then, if $i=\liminf \int_{G} \eta_{k}(t) d t$ and $\eta(t)=f_{0}(t, x(t), u(t)), t \in G$, we have $\eta \in L_{1}(G)$ and $\int_{G} \eta(t) d t \leqslant i$.

(b) The same as (a), where $f_{0}(t, x, u)$ satisfies condition $\left(\mathrm{G}_{p q}^{*}\right)$ for some $1 \leqslant p, q \leqslant \infty$, and where $x, x_{k} \in\left(L_{p}(G)\right)^{n}, u, u_{k} \in\left(L_{q}(G)\right)^{m}$, $\eta_{k} \in L_{1}(G),\|x\|_{p},\left\|x_{k}\right\|_{p} \leqslant L_{0},\|u\|_{q},\left\|u_{k}\right\|_{q} \leqslant L, x_{k}(t) \rightarrow x(t)$ in measure in $G, u_{k} \rightarrow u$ weakly in $\left(L_{1}(G)\right)^{m}$, and $-\infty<i<+\infty$.

(c) The same as (a) where $f_{0}(t, x, u)$ satisfies condition $\left(H_{q}{ }^{*}\right)$ with $\phi(u)=u$ for some $1 \leqslant q \leqslant \infty$, where the functions $x, x_{k}$ are only measurable, $u, u_{k} \in\left(L_{q}(G)\right)^{m},\|u\|_{q},\left\|u_{k}\right\|_{q} \leqslant L, u_{k} \rightarrow u$ weakly in $\left(L_{1}(G)\right)^{m}$ and $x_{k}(t) \rightarrow x(t)$ in measure as $k \rightarrow \infty$.

Proof. Let us prove (b). By (C) the sets $A(t)$ are closed for almost all $t \in G$. Since $x_{i}(t) \in A(t), t \in G$, and for a suitable subsequence $x_{k_{s}}(t) \rightarrow x(t)$ pointwise almost everywhere in $G$, we conclude that $x(t) \in A(t)$ a.e. in $G$. Also, $u_{k}(t) \in U(t), t \in G$, where the sets $U(t)$ are convex for almost all $t \in G$. By property (C) the same sets $U(t)$ are also closed for almost all $t \in G$. Since $u_{k} \rightarrow u$ weakly in $\left(L_{q}(G)\right)^{m}$, by force of Statement 5.1 we have $u(t) \in U(t)$ a.e. in $G$. Let $C=\int_{G} \psi(t) d t$, and let $L_{0}, L$ be constants such that $\left\|x_{k}\right\|_{p} \leqslant L_{0},\left\|u_{k}\right\|_{q} \leqslant L,\|x\|_{p} \leqslant L_{0}$, $\|u\|_{q} \leqslant L$. Note that

$$
\left|\eta_{k}(t)\right|=\left|f_{0}\left(t, x_{k}(t), u_{k}(t)\right)\right| \leqslant \psi(t)+c\left|x_{k}(t)\right|^{p}+c^{\prime}\left|u_{k}(t)\right|^{q},
$$

and an analogous relation holds for $\eta$. Thus $\eta, \eta_{k} \in L_{1}(G)$, and $\|\eta\|_{1}$, $\left\|\eta_{k}\right\|_{1} \leqslant C+c L_{0}{ }^{p}+c^{\prime} L^{q}$, and certainly $i$ is finite.

From Statement 3.1, $f_{0}$ has property $(\mathscr{D})$, so that, if $\bar{\eta}_{k}(t)=$ $f_{0}\left(t, x(t), x_{k}(t)\right), t \in G$, and $\delta_{k}(t)=\eta_{k}(t)-\bar{\eta}_{k}(t)$, then $\delta_{k} \rightarrow 0$ strongly in $L_{1}(G)$ as $k \rightarrow \infty$. Let $j_{k}=\int_{G} \eta_{k}(t) d t, j_{k}{ }^{\prime}=\int_{G} \bar{\eta}_{k}(t) d t$. We may well assume, taking a suitable subsequence, that $j_{k} \rightarrow i$ as $k \rightarrow \infty$. Then we 
have also $j_{k}{ }^{\prime} \rightarrow i$ as $k \rightarrow \infty$. Here $u_{k} \rightarrow u$ weakly in $\left(L_{q}(G)\right)^{m}$. By BanachSaks-Mazur theorem (see Ref. 12 and Ref. 13, page 46), there are numbers $c_{N k} \geqslant 0, k=1, \ldots, N, N=1,2, \ldots$, with $\sum_{k=1}^{N} c_{N k}=1$, such that, if $v_{N}(t)=\sum_{k=1}^{N} c_{N k} u_{k}(t), t \in G$, then $v_{N} \rightarrow u$ strongly in $\left(L_{q}(G)\right)^{m}$. Since the sets $U(t)$ are convex we have $v_{N}(t) \in U(t), t \in G$ (a.e.), $N=$ $1,2, \ldots$ We can choose the sequence $c_{N k}$ in such a way that we have also $\sum_{k=1}^{N} c_{N k} j_{k}{ }^{\prime} \rightarrow i$ as $N \rightarrow \infty$. [Indeed, $j_{k}{ }^{\prime} \rightarrow i$, and we apply Mazur theorem to the product space $\left(L_{q}(G)\right)^{m} \times E_{1}$.] We consider now the operation from functions $u(t), t \in G, u \in\left(L_{q}(G)\right)^{m}$ to the functions $f_{0}(t, x(t), u(t))=$ $(\mathscr{F} u)(t), t \in G$, as defining a Carathéodory operator $\mathscr{F}:\left(L_{q}(G)\right)^{m} \rightarrow L_{\mathbf{1}}(G)$. Since $v_{N} \rightarrow u$ strongly in $\left(L_{q}(G)\right)^{m}$, we know (Ref. 5, page 22, no. 2) that $f_{0}\left(t, x(t), v_{N}(t)\right) \rightarrow f_{0}(t, x(t), u(t))$ strongly in $L_{1}(G)$ as $N \rightarrow \infty$. On the other hand, because of the convexity of $f_{0}$, we have

Hence,

$$
f_{0}\left(t, x(t), v_{N}(t)\right)=f_{0}\left(t, x(t), \sum_{k=1}^{N} c_{N k} u_{k}(t)\right) \leqslant \sum_{k=1}^{N} c_{N k} f_{0}\left(t, x(t), u_{k}(t)\right) .
$$

$$
\begin{aligned}
\int_{G} \eta(t) d t & =\int_{G} f_{0}(t, x(t), u(t)) d t=\lim _{k \rightarrow \infty} \int_{G} f_{0}\left(t, x(t), v_{k}(t)\right) d t \\
& \leqslant \liminf _{N \rightarrow \infty} \sum_{k=1}^{N} c_{N k} \int_{G} f_{0}\left(t, x(t), u_{k}(t)\right) d t \\
& =\lim _{N \rightarrow \infty} \sum_{k=1}^{N} c_{N k} j_{k}{ }^{\prime}=i .
\end{aligned}
$$

Part (b) of Theorem 10.1 is thereby proved. The proofs of parts (a) and (c) are analogous.

Remark 10.1. The above proof can be easily modified in the case where $\phi(u)$ is an arbitrary continuous function with $|\phi(u)| \rightarrow \infty$ as $|u| \rightarrow \infty$.

Remark 10,2. The following simple lower semicontinuity theorem was proved by Cesari in Refs. 3, 4 for the case $\nu=1$ and integrals of the form

$$
I[x]=\int_{t_{1}}^{t_{2}} f_{0}\left(t, x(t), x^{\prime}(t)\right) d t
$$

Theorem 10.2, Let $A$ be a closed subset of the $t x$-space $E^{n+1}$, $\left(t \in E^{1}, x=\left(x^{1}, \ldots, x^{n}\right) \in E^{n}\right)$, and let $f_{0}(t, x, u)$ be a real-valued function which is continuous in $A \times E^{n}$, and convex in $u$ for every $(t, x) \in A$. If $x(t), x_{k}(t), t_{1} \leqslant t \leqslant t_{2}, k=1,2, \ldots$, are absolutely continuous functions, 
$\left(t, x_{k}(t)\right) \in A$, such that $x_{k} \rightarrow x$ uniformly in $\left[t_{1}, t_{2}\right]$ and $x_{k}{ }^{\prime} \rightarrow x^{\prime}$ weakly in $\left(L_{1}\left(\left[t_{1}, t_{2}\right]\right)\right)^{n}$ as $k \rightarrow \infty$, then $(t, x(t)) \in A$ and $I[x] \leqslant \lim _{\text {inf }} k_{k \rightarrow \infty} I\left[x_{k}\right]$, where each of these integrals necessarily is finite, or $+\infty$.

Also, corresponding semicontinuity theorems for multiple integrals $(\nu>1)$, and closure and lower closure theorems for Mayer and Lagrange problems are found in Ref. 3.

\section{References}

1. Cesari, L., Closure Theorems for Orientor Fields and Weak Convergence, Archive for Rational Mechanics and Analysis, Vol. 55, pp. 332-356, 1974.

2. Cesari, L., Closure Theorems for Orientor Fields, Bulletin of the American Mathematical Society, Vol. 79, pp. 684-689, 1973.

3. CESARI, L,, Lower Semicontinuity and Lower Closure Theorems Without Semninormality Condition, Annali di Matematica Pura e Applicata, Vol. 98, pp. 381-397, 1974.

4. CESARI, L., A Necessary and Sufficient Condition for Lower Semicontinuity, Bulletin of American Mathematical Society, Vol. 80, pp. 467-472, 1974.

5. Krasnoselskir, M. A., Topological Methods in the Theory of Nonlinear Integral Equations, The Macmillan Company, New York, New York, 1964.

6. Cesari, L., and Suryanarayana, M. B., Convexity and Property $(Q)$ in Optimal Control Theory, SIAM Journal on Control, Vol. 12, pp. 705-720, 1974.

7. Dunford, N., and Schwartz, J. T., Linear Operators, $I$, John Wiley and Sons (Interscience Publishers), New York, New York, 1958.

8. BRowDER, F. E., Remarks on the Direct Method of the Calculus of Variations, Archive for Rational Mechanics and Analysis, Vol. 20, pp. 251-258, 1965.

9. Rothe, E. H., An Existence Theorem in the Calculus of Variations, Archive for Rational Mechanics and Analysis, Vol. 21, pp. 151-162, 1966.

10. Morrey, C. B., Multiple Integrals in the Calculus of Variations, SpringerVerlag, New York, New York, 1966.

11. MCShane, E. J., and Warfreld, R. B., On Filippov's Implicit Function Lemma, Proceedings of the American Mathematical Society, Vol. 18, pp. 41-47, 1967.

12. MazUR, S., Über Konvexe Mengen in Linearen Normierten Raumen, Studia Mathematica, Vol. 4, pp. 70-84, 1933.

13. DAY, M. M., Functional Analysis in Normed Spaces, Springer-Verlag, New York, New York, 1973.

14. Krasnoselski, M. A., Zabreiko, P. R., Pustyl'nik, E. I., and Sobolevskir, P. W., Integral Operators in Spaces of Summable Functions (in Russian), Nauka, Moscow, USSR, 1966. 